\title{
STOCHASTIC CONTROL PROBLEMS AND SPHERICAL FUNCTIONS ON SYMMETRIC SPACES
}

\author{
T. E. DUNCAN AND H. UPMEIER
}

\begin{abstract}
A family of explicitly solvable stochastic control problems is formulated and solved in noncompact symmetric spaces. The symmetric spaces include all of the classical spaces and four of the exceptional spaces. The stochastic control problems are the control of Brownian motion in these symmetric spaces by a drift vector field. For each symmetric space a family of stochastic control problems is formulated by using spherical functions in the cost functionals. These spherical functions are explicitly described and are polynomials in suitable coordinates. A generalization to abstract root systems is given.
\end{abstract}

\section{INTRODUCTION}

In the investigation of stochastic optimal control for nonlinear stochastic systems it is natural to study the optimal control of diffusion processes in a Riemannian manifold. Some sufficient, as well as necessary and sufficient, conditions have been obtained for the optimal controls for such controlled diffusions. The first results for these problems are given in [D2, D3]. A natural controlled diffusion in a manifold can be formed from a stochastic differential equation that is the sum of the formal vectors of a manifold-valued Brownian motion and a drift vector field that depends on the control. For many reasons it is important to have examples of such controlled diffusions where the optimal control is expressed explicitly as a function of the state of the controlled diffusion. However even in Euclidean space there are only a relatively few examples of controlled diffusions where the optimal control is expressed explicitly as a function of the state.

In the more general setting of Riemannian geometry a natural class of (curved) manifolds are the symmetric spaces which are closely related to the semisimple and reductive Lie groups and arise in many classical situations (e.g. matrix groups and hyperbolic spaces). By using the additional structure available on symmetric spaces we are able to construct large families of explicitly solvable stochastic control problems in symmetric spaces of arbitrary rank.

Received by the editors October 26, 1992.

1991 Mathematics Subject Classification. Primary 93E20, 43A90; Secondary 58G32, 33C55, $53 \mathrm{C} 35$.

Key words and phrases. Stochastic control, spherical functions, controlled diffusions in symmetric spaces, Laplace-Beltrami operator, explicitly solvable control problems.

The first author was supported in part by NSF grant ECS-8718026, and the second was supported in part by NSF grant DMS-8702371. 
The first example of an explicitly solvable stochastic control problem in a noncompact manifold with nonzero curvature is a controlled diffusion in real hyperbolic three space [D4]. This example in real hyperbolic three space is generalized in [D5] to all the hyperbolic spaces, that is, irreducible noncompact symmetric spaces of rank one, and a countable family of nontrivially distinct cost functionals for each of these spaces. In all of these examples some spherical functions, that are polynomials in appropriate variables, are used to define the cost functions. For rank one symmetric spaces these spherical functions are hypergeometric functions. The stochastic control problem is to control Brownian motion in a hyperbolic space by a drift vector field so that it remains close to a fixed point in the space called the origin. The cost functionals are positive, monotone increasing functions of the distance of the state from the origin and are quadratic functions of the control.

In this paper a family of stochastic control problems are formulated and explicitly solved in noncompact symmetric spaces of arbitrary rank that are formed from semisimple and reductive Lie groups. Specifically all symmetric cones and (complex) symmetric balls are used. The stochastic control problem is the natural analogue of the stochastic control problems in the hyperbolic spaces solved in [D4, D5]. The problem is to control Brownian motion in a noncompact symmetric space by a drift so that it remains close to the origin. Spherical functions are used to construct a family of cost functionals. For the symmetric cones spherical polynomials have been constructed in the statistics literature (e.g. [M2]). In this paper these spherical polynomials are constructed more easily from the representation theory of the symmetric cones [U2]. For symmetric balls not much is known about a general construction of spherical polynomials [D1, H1]. In this paper an explicit procedure is given for the construction of all spherical polynomials in an arbitrary symmetric ball. This procedure uses the spherical polynomials in symmetric cones. These spherical polynomials are monotone increasing along rays in a positive Weyl chamber. An admissible control is required to induce a stochastic differential equation for the controlled diffusion that has a unique strong solution. The solution of each of these stochastic control problems is obtained by finding a smooth solution to the Hamilton-Jacobi or dynamic programming equation for the control problem.

The organization of this paper is as follows. In Sections 2, 3, 4, 7 and 8, we develop the relevant geometric features for the symmetric cones and symmetric balls, respectively. We start from one basic fact, namely the Jordan-theoretic description of these two classes of symmetric spaces and their infinitesimal isometries [L3, U1, FK], and develop the root decomposition, the structure of the Laplace operator in radial coordinates, and the basic properties of spherical functions in a coherent way. This makes the paper more self-contained and underlines the close analogy between (real) symmetric cones and (complex) symmetric balls. (A more comprehensive account of the Jordan-theoretic description of symmetric spaces, which so far is not readily available in the literature, can be found in a forthcoming book [FK].) In Sections 5, 6, and 9, these geometric results are then used in the study of the corresponding stochastic control problem. Finally, Section 10 indicates how these results can be generalized to the so-called abstract root systems [D1], replacing geometric arguments by combinatorial properties of the spherical polynomials. 


\section{REVIEW OF STOCHASTIC CALCULUS}

Stochastic optimal control of a diffusion process in a Riemannian manifold is based on the stochastic calculus of Brownian motion. This stochastic calculus is an interplay between the properties of a Brownian motion in a Euclidean space and the theory of smooth curves in a Riemannian manifold. To make this paper accessible to an audience including those without a background in probability, some of the basic concepts and approaches of stochastic calculus and control in manifolds are briefly reviewed. A Brownian motion in a Riemannian manifold can be defined in various ways. Assume first that $X$ is a measurable space. Let $B(X)$ be the Banach algebra of all bounded measurable functions $f: X \rightarrow \mathbb{C}$, endowed with the norm

$$
\|f\|:=\sup _{x \in X}|f(x)| .
$$

Let $B^{\prime}(X)$ be the space of all complex measures $\mu$ on $X$ such that

$$
\|\mu\|:=\int_{X}|\mu|(d x)<\infty .
$$

Via the natural pairing

$$
\langle\mu, f\rangle:=\int_{X} \mu(d x) f(x),
$$

$B^{\prime}(X)$ becomes a (proper) subspace of the topological dual space of $B(X)$.

Let $Y$ be another measurable space. A mapping $K: X \rightarrow B^{\prime}(Y)$, denoted by $x \mapsto K(x, d y)$, is called a measure kernel if, for every measurable subset $B \subset Y$, the mapping

$$
X \ni x \mapsto \int_{B} K(x, d y)
$$

is measurable, and we have

$$
\|K\|:=\sup _{x \in X} \int_{Y}|K(x, d y)|<\infty .
$$

$K$ can be identified with a continuous linear operator $B(X) \stackrel{K}{\longleftarrow} B(Y)$, defined by

$$
(K f)(x):=\int_{Y} K(x, d y) f(y)
$$

for all $f \in B(Y)$ and $x \in X$. If $L: Y \rightarrow B^{\prime}(Z)$ is another measure kernel, we may define

$$
(K L)(x, d z):=\int_{Y} K(x, d y) L(y, d z)
$$

and obtain a measure kernel $K L: X \rightarrow B^{\prime}(Z)$ since we have, by Fubini's Theorem,

$$
\int_{Z}|(K L)(x, d z)| \leq \int_{Y}|K(x, d y)| \int_{Z}|L(y, d z)|
$$

for all $x \in X$, showing that $\|K L\| \leq\|K\|\|L\|$. As an operator, $K L$ is the composition of $K$ and $L$.

Let $B_{1}^{\prime}(X)$ be the subset of $B^{\prime}(X)$ consisting of all (positive) probability measures on $X$; i.e., $|\mu|=\mu$ and $\|\mu\|=1$ for $\mu \in B_{1}^{\prime}(X)$. 
Put $T:=\{t \in \mathbb{R}: t \geq 0\}$, and consider a family

$$
K_{t}: X \rightarrow B_{1}^{\prime}(X) \quad(t \in T)
$$

of measure kernels satisfying $K_{s} K_{t}=K_{s+t}$ for all $s, t \in T$. For any subset $I \subset T$, consider the direct product measurable space

$$
X_{I}:=\prod_{i \in I} X
$$

endowed with the $\sigma$-algebra of cylinder sets. For $I \subset \mathscr{I} \subset T$, we have a canonical projection $p_{I}^{\mathscr{I}}: X_{\mathscr{I}} \rightarrow X_{I}$. For any finite subset $I=\left\{t_{1}<\cdots<t_{n}\right\}$ of $T$, there exists a measure kernel $K_{I}: X \rightarrow B_{1}^{\prime}\left(X_{I}\right)$ uniquely determined by the property

$$
\begin{aligned}
K_{I}\left(x_{0}, \prod_{i \in I} B_{i}\right):= & \int_{B_{t_{1}}} K_{t_{1}-t_{0}}\left(x_{0}, d x_{1}\right) \\
& \cdot \int_{B_{t_{2}}} K_{t_{2}-t_{1}}\left(x_{1}, d x_{2}\right) \cdots \int_{B_{t_{n}}} K_{t_{n}-t_{n-1}}\left(x_{n-1}, d x_{n}\right)
\end{aligned}
$$

for all $x_{0} \in X$ and all measurable subsets $B_{i} \subset X(i \in I)$. Here we put $t_{0}:=0$. If $\mathscr{I} \supset I$ is another finite subset of $T$, there is a commuting diagram

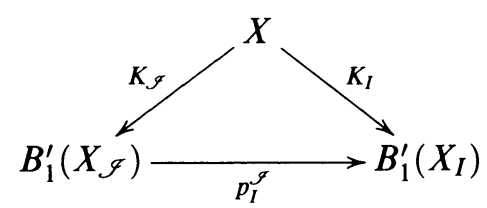

where the horizontal arrow is the direct image map under $p_{I}^{\mathscr{I}}$. Thus $\left(K_{I}\right)$ is a projective system of measure kernels and there exists a measure kernel

$$
K_{T}: X \rightarrow B_{1}^{\prime}\left(X_{T}\right)
$$

such that for every finite subset $I \subset T$ the diagram

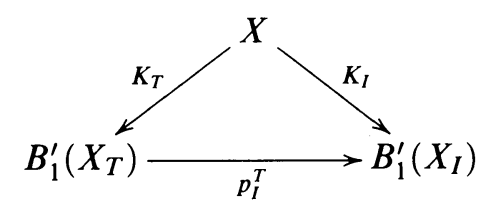

commutes. $K_{T}$ is called the projective limit associated with the semigroup (1.1).

An important class of semigroups of measure kernels comes from elliptic differential operators. Let $X$ be a locally compact smooth manifold and let $\mathscr{E}(X)$ be the Fréchet algebra of all smooth functions $f: X \rightarrow \mathbb{C}$, endowed with the seminorms

$$
|f|_{D, K}:=\sup _{x \in K}|(D f)(x)|
$$

where $K \subset X$ is compact and $D$ is a differential operator with smooth coefficients on $X$.

Let $\mathscr{E}^{\prime}(X)$ be the topological dual space of $\mathscr{E}(X)$, consisting of all distributions $\delta$ on $X$ with compact support. The natural pairing between $\mathscr{E}(X)$ and $\mathscr{E}^{\prime}(X)$ is denoted by

$$
\langle\delta, f\rangle=: \int_{X} \delta(d x) f(x) .
$$


If $Y$ is another smooth manifold, a mapping $D: X \rightarrow \mathscr{E}^{\prime}(Y)$, denoted by $x \mapsto D(x, d y)$, is called a distribution kernel if for every $f \in \mathscr{E}(Y)$ the function

$$
(D f)(x):=\int_{Y} D(x, d y) f(y)
$$

is smooth on $X$, and defines a continuous operator $\mathscr{E}(X) \stackrel{D}{\longleftarrow} \mathscr{E}(y)$. If $E: Y \rightarrow$ $\mathscr{E}^{\prime}(Z)$ is another distribution kernel, the continuity assumption implies that

$$
(D E)(x, d z):=\int_{Y} D(x, d y) E(y, d z)
$$

defines a distribution kernel $D E: X \rightarrow \mathscr{E}^{\prime}(Z)$ corresponding to the composition of operators. Any differential operator $\mathscr{E}(X) \stackrel{D}{\longleftarrow} \mathscr{E}(X)$ is continuous and can be identified with a distribution kernel

$$
\int_{X} D(x, d y) f(y):=(D f)(x)
$$

from $X$ to $\mathscr{E}^{\prime}(X)$. For each $x, D(x, d y)$ has compact support $\subset\{x\}$.

If $D$ is a second-order differential operator which is strictly elliptic in the sense of [Y2, p. 419 and p. 426], there exists a fundamental solution which is a semigroup of measure kernels

$$
\exp _{t} D: X \rightarrow B_{1}^{\prime}(X) \quad(t \in T)
$$

(or of operators on $B(X)$ ). According to (1.2), we obtain a measure kernel

$$
\exp _{T} D: X \rightarrow B_{1}^{\prime}\left(X_{T}\right) \text {. }
$$

We now consider the important special case $X=\mathbb{R}^{n}$ and the algebra $B^{\prime}\left(\mathbb{R}^{n}\right)$ of all bounded complex Borel measures, with convolution

$$
\int_{\mathbf{R}^{n}}\left(\mu^{*} \nu\right)(d z) f(z)=\int_{\mathbf{R}^{n}} \int_{\mathbf{R}^{n}} \mu(d x) \nu(d y) f(x+y) .
$$

Every $\mu \in B^{\prime}\left(\mathbb{R}^{n}\right)$ defines a measure kernel $\mu^{\#}: \mathbb{R}^{n} \rightarrow B^{\prime}\left(\mathbb{R}^{n}\right)$, denoted by $x \mapsto \mu^{\#}(x, d y)$, via the formula $\mu^{\#}(x, B):=\mu(B-x)$ for all Borel subsets $B \subset \mathbb{R}^{n}$. The associated convolution operator

$$
\left(\mu^{\#} f\right)(x)=\int_{\mathbf{R}^{n}} \mu^{\#}(x, d y) f(y)=\int_{\mathbf{R}^{n}} \mu(d y) f(x+y)
$$

commutes with translations, and we have

$$
\mu^{\#} \nu^{*}=\left(\mu^{*} \nu\right)^{*}
$$

for the product of measure kernels. Now consider a family $\mu_{t} \in B_{1}^{\prime}\left(\mathbb{R}^{n}\right)$ of probability measures, for $t \in T:=\mathbb{R}_{+}$, satisfying

$$
\mu_{s}^{\#} \mu_{t}=\mu_{s+t}
$$

for all $s, t \in T$. For each finite subset $I=\left\{t_{1}<t_{2}<\cdots<t_{n}\right\}$ of $T$ define a measure $\mu_{I} \in B_{1}^{\prime}\left(\mathbb{R}_{I}^{n}\right)$ by putting

$$
\begin{aligned}
\mu_{I}\left(\prod_{i \in I} B_{i}\right):= & \int_{B_{t_{1}}} \mu_{t_{1}-t_{0}}\left(d x_{1}\right) \int_{B_{t_{2}}-x_{1}} \mu_{t_{2}-t_{1}}\left(d x_{2}\right) \\
& \cdots \int_{B_{t_{n}}-x_{1}-\cdots-x_{n-1}} \mu_{t_{n}-t_{n-1}}\left(d x_{n}\right)
\end{aligned}
$$


for all Borel measurable subsets $B_{i} \subset \mathbb{R}^{n}(i \in I)$. Here we put $t_{0}:=0$.

If $\mathscr{I} \supset I$ is another finite subset of $T$, the canonical projection gives rise to the direct image $\mu_{\mathscr{I}} \rtimes p_{I}^{\mathscr{J}}=\mu_{I}$. Thus we obtain a projective system $\left(\mu_{I}\right)$ of measures and there exists a measure $\mu_{T} \in B_{1}^{\prime}\left(\mathbb{R}_{T}^{n}\right)$ such that

$$
\mu_{T} \rtimes p_{I}^{T}=\mu_{I} \quad(I \subset T \text { finite }) .
$$

The measure $\mu_{T}$ is called is called the projective limit associated with the semigroup (1.5). According to (1.4), the associated measure kernels

$$
K_{t}=\mu_{t}^{\#}: \mathbb{R}^{n} \rightarrow B_{1}^{\prime}\left(\mathbb{R}^{n}\right)
$$

form a semigroup as well, and we have

$$
K_{I}=\mu_{I}^{\#} \quad(I \subset T \text { finite }) \text { and } K_{T}=\mu_{T}^{\#} .
$$

An important class of semigroups of measures comes from constant-coefficient differential operators. Consider the algebra $\mathscr{E}_{0}^{\prime}\left(\mathbb{R}^{n}\right)$ of all distributions $\delta$ on $\mathbb{R}^{n}$ supported at $\{0\}$, with convolution

$$
\int_{\mathbb{R}^{n}}\left(\delta^{\#} \varepsilon\right)(d z) f(z)=\int_{\mathbb{R}^{n}} \int_{\mathbb{R}^{n}} \delta(d x) \varepsilon(d y) f(x+y)
$$

for all $f \in \mathscr{E}\left(\mathbb{R}^{n}\right)$. Every $\delta \in \mathscr{E}_{0}^{\prime}\left(\mathbb{R}^{n}\right)$ defines a distribution kernel $\delta^{\#}: \mathbb{R}^{n} \rightarrow$ $\mathscr{E}^{\prime}\left(\mathbb{R}^{n}\right)$, denoted by $x \mapsto \delta^{\#}(x, d y)$, via the formula

$$
\left(\delta^{\#} f\right)(x)=\int_{\mathbb{R}^{n}} \delta^{\#}(x, d y) f(y):=\int_{\mathbb{R}^{n}} \delta(d y) f(x+y)
$$

for all $f \in \mathscr{E}\left(\mathbb{R}^{n}\right)$. We have $\delta^{\#} \varepsilon^{\#}=\left(\delta^{\#} \varepsilon\right)^{\#}$ for the product of distribution kernels. Any constant-coefficient differential operator

$$
\delta=\sum_{\alpha} c_{\alpha}\left(\frac{\partial}{\partial x_{1}}\right)^{\alpha_{1}} \cdots\left(\frac{\partial}{\partial x_{n}}\right)^{\alpha_{n}}
$$

can be identified with the distribution

$$
\int_{\mathbb{R}^{n}} \delta(d x) f(x)=(\delta f)(0)
$$

in $\mathscr{E}_{0}^{\prime}\left(\mathbb{R}^{n}\right)$. By translation invariance, $\delta$ induces a differential operator

$$
\mathscr{E}\left(\mathbb{R}^{n}\right) \stackrel{\delta^{*}}{\longleftarrow} \mathscr{E}\left(\mathbb{R}^{n}\right)
$$

with distribution kernel defined by (1.6).

In case $\delta$ is strictly elliptic of second order [H3] there exists a fundamental solution which is a semigroup of measures

$$
\exp _{t} \delta \in B_{1}^{\prime}\left(\mathbb{R}^{n}\right) \quad(t \in T)
$$

whose associated measure kernels

$$
\left(\exp _{t} \delta\right)^{\#}=\exp _{t} \delta^{\#}: \mathbb{R}^{n} \rightarrow B_{1}^{\prime}\left(\mathbb{R}^{n}\right)
$$

form the fundamental solution of the elliptic operator (1.7). According to (1.3), we obtain a measure

$$
\exp _{T} \delta \in B_{1}^{\prime}\left(\mathbb{R}_{T}^{n}\right)
$$


Every Riemannian manifold $X$ has a canonical elliptic second-order differential operator $\Delta$ called the Laplace-Beltrami operator. The associated measure kernel

$$
\exp _{T} \Delta: X \rightarrow B_{1}^{\prime}\left(X_{T}\right)
$$

(cf. (1.3)) is the transition probability of the Markov process called the Brownian motion on $X$. One can show [IW] that, for every $x \in X$, the probability measure $\left(\exp _{T} \Delta\right)(x, d y)$ has support on the continuous paths $\mathscr{C}(T, X) \subset X_{T}$. Thus we have

$$
\exp _{T} \Delta: X \rightarrow B_{1}^{\prime}(\mathscr{C}(T, X)) .
$$

If $X=\mathbb{R}^{n}$ is Euclidean space and

$$
\delta:=\sum_{i=1}^{n} \frac{\partial^{2}}{\partial x_{i}^{2}}
$$

is the (strictly elliptic) Casimir operator at 0, the Laplace-Beltrami operator is $\Delta=\delta^{\#}$ and we have

$$
\left(\exp _{t} \delta\right)(d x)=(4 \pi t)^{-n / 2} e^{-|x|^{2} / 4 t} d x
$$

for all $t \in T$. The associated measure

$$
\exp _{T} \delta \in B_{1}^{\prime}\left(\mathbb{R}_{T}^{n}\right)
$$

(cf. (1.8)) is called the transition probability of the Brownian motion at $0 \in \mathbb{R}^{n}$. One can show [IW] that the probability measure (1.11) has support on the continuous paths $\mathscr{C}\left(T, \mathbb{R}^{n}\right) \subset \mathbb{R}_{T}^{n}$, so that

$$
\exp _{T} \delta \in B_{1}^{\prime}\left(\mathscr{C}\left(T, \mathbb{R}^{n}\right)\right) \text {. }
$$

The two concepts (1.10) and (1.11) are related via the geodesic coordinates

$$
\Omega_{x} \stackrel{\exp _{x}}{\longrightarrow} X
$$

at any "base point" $x \in X$, assigning to a tangent vector $v$ in a 0 -neighborhood $\Omega_{x} \subset T_{x} X$ the geodesic $t \mapsto \exp _{x}(t v)$ passing through $x$ in direction $v$. More precisely, $\left(\exp _{T} \Delta\right)(x, d y)$ can locally be described as the image measure of $\left(\exp _{T} \delta\right)(d y)$ under the local diffeomorphism (1.12). Here $\delta$ is the Casimir operator on $T_{x} X=\mathbb{R}^{n}$.

A sample path approach to the construction of a Brownian motion in a Riemannian manifold $M$ can be made using the inverse of the development of É. Cartan that acts on the family of piecewise smooth curves in the manifold with the same initial point $p$. More generally or abstractly the development (of É. Cartan) and its inverse define a diffeomorphism between the Sobolev space of absolutely continuous functions in the tangent space $T_{p} M$ starting at the origin whose derivatives are square integrable and the Sobolev manifold of absolutely continuous $M$-valued functions whose derivatives are square integrable with respect to the Riemannian metric. Actually this construction of Brownian motion cannot be extended to all noncompact, complete Riemannian manifolds. It is necessary to put some conditions on the curvature. The curvature conditions ensure that the Brownian motion does not have a finite escape time. With this construction of Brownian motion, many of the results for a Brownian 
motion in Euclidean space can be directly translated to the manifold $M$ using the horizontal lift of Brownian motion to the bundle of orthonormal frames, $O(M)$.

In analogy with Euclidean space a stochastic optimal control problem in a Riemannian manifold $M$ is described by the local description of a controlled diffusion process (that is, a stochastic differential equation) and a cost functional. The problem is to choose a control from some family of admissible controls that depends on the current positions (or past history) of the process so that the cost functional is minimized. An important method for the minimization problem is to find a smooth solution of the associated Hamilton-Jacobi equation and verify that the control obtained by the procedure is admissible. In the problems considered here the Riemannian manifold is a noncompact symmetric space. The controlled diffusion is Brownian motion with a control as the drift vector. The cost functional is the sum of a quadratic function of the control and a spherical function so it is desired to keep the diffusion close to the "origin" of the symmetric space. Since the spherical functions and the controlled diffusions have some group invariance properties the solution of the control problem is determined from the behavior of the controlled diffusion in a subspace of the symmetric space.

\section{SYMMETRIC SPACES AND JORDAN TRIPLES}

We now introduce the "phase spaces" for our stochastic control problems, the symmetric spaces of arbitrary rank (for rank 1, cf. [D4]). The non-Euclidean symmetric spaces fall into two dual classes, the compact type and the noncompact type which have a simpler topology (no antipodal points, no closed geodesics, etc.) but on the other hand exhibit a highly nontrivial (stratified) boundary structure. It is known [L1] that a Riemannian symmetric space is algebraically characterized by a ternary product structure (Lie triple system) $[x y z]$ on its tangent space $Z$ at an arbitrary base point. This triple system is given by the curvature tensor. More important to us is another ternary product (Jordan triple system) $\left\{x y^{*} z\right\}$ on $Z$ which is related to the Lie triple via

$$
[x y z]=\left\{x y^{*} z\right\}-\left\{y x^{*} z\right\} \text {. }
$$

Thus the Jordan triple allows for the decomposition of $[x y z]$ into "positive" and "negative" part. The symmetric spaces admitting such a Jordan triple structure are called symmetric $R$-spaces [L2]; they include all classical spaces and 4 exceptional types.

We will now indicate how Jordan triple systems can be used to give an explicit and uniform construction for

(i) the noncompact model of the symmetric $R$-space as an unbounded domain in $Z$;

(ii) the semisimple Lie group $G$ (or rather its Lie algebra $\mathfrak{g}$ ) of isometries; $\Delta$.

(iii) conical and spherical eigenfunctions of the Laplace-Beltrami operator

A Jordan triple product on $Z$ is a trilinear map

$$
Z \times Z \times Z \ni(x, y, z) \mapsto\left\{x y^{*} z\right\} \in Z
$$

satisfying $\left\{x y^{*} z\right\}=\left\{z y^{*} x\right\}$ (commutativity) and the commutator identity

$$
\left[u \square v^{*}, x \square y^{*}\right]=\left\{u v^{*} x\right\} \square y^{*}-x \square\left\{y u^{*} v\right\}^{*}
$$


for the linear endomorphisms $\left(x \square y^{*}\right) z:=\left\{x y^{*} z\right\}$ on $Z$. We assume in addition that the bilinear form $(x, y) \mapsto$ trace $x \square y^{*}$ is symmetric and positive definite.

We denote vector fields on $Z$ by the symbol $f(z) \frac{\partial}{\partial z}$ where $z \in Z$ is a "variable" and $f: Z \rightarrow Z$ is smooth. The commutator is

$$
\left[f_{1}(z) \frac{\partial}{\partial z}, f_{2}(z) \frac{\partial}{\partial z}\right]=\left(d f_{1}(z) f_{2}(z)-d f_{2}(z) f_{1}(z)\right) \frac{\partial}{\partial z}
$$

where $d f$ is the derivative.

For example, every linear endomorphism $M: Z \rightarrow Z$ gives rise to a vector field $M z \frac{\partial}{\partial z}$ and we have

$$
\left[M_{1} z \frac{\partial}{\partial z}, M_{2} z \frac{\partial}{\partial z}\right]=\left[M_{1}, M_{2}\right] z \frac{\partial}{\partial z} .
$$

In particular, we have the linear vector fields $\left\{x y^{*} z\right\} \frac{\partial}{\partial z}(x, y \in Z$ fixed) which, by (2.1) and (2.2), form a Lie algebra $l^{0}$. Moreover, the "Jordan triple identity" (2.2) implies that the "constant" vector fields

$$
\mathfrak{l}^{1}=\left\{a \frac{\partial}{\partial z}: a \in Z\right\}
$$

and the "quadratic" vector fields

$$
\mathfrak{l}^{-1}=\left\{\left\{z a^{*} z\right\} \frac{\partial}{\partial z}: a \in Z\right\}
$$

give rise to a Lie algebra

$$
\mathfrak{l}=\mathfrak{l}^{1} \oplus \mathfrak{l}^{0} \oplus \mathfrak{l}^{-1}
$$

which, by [L2, Theorem 1], is semisimple. Let $L$ be the corresponding centerfree connected lie group. Then the normalizer $P$ of $\mathfrak{l}^{1}$ is a parabolic subgroup and $L / P$ is a compact symmetric $R$-space.

We are interested in the noncompact dual space of $L / P$ which can always be be realized as a symmetric domain (either bounded or unbounded) in $Z$. Realized as a bounded domain $\Omega$, a transitive (reductive) Lie group of isometries has a Lie algebra $\mathfrak{g}$ with Cartan decomposition $\mathfrak{g}=\mathfrak{l} \oplus \mathfrak{p}$ where

$$
\mathfrak{l}=\left\langle\left(\left\{a b^{*} z\right\}-\left\{b a^{*} z\right\}\right) \frac{\partial}{\partial z}: a, b \in Z\right\rangle
$$

and

$$
\mathfrak{p}=\left\{\left(a-\left\{z a^{*} z\right\}\right) \frac{\partial}{\partial z}: a \in Z\right\} .
$$

More appropriate for our purposes is the unbounded realization $\Pi$ as a "Siegel domain". In order to construct $\Pi$, we need the notion of the Cayley transformation [KW, L3] associated with an element $e \in Z$ satisfying the "tripotent" condition $\left\{e e^{*} e\right\}=e$. The Cayley transform is the analytic automorphism

$$
g_{e}=\exp \left(\frac{\pi}{4}\left(e+\left\{z e^{*} z\right\}\right) \frac{\partial}{\partial z}\right)
$$

of $L / P$. Note that its defining vector field belongs to $\mathfrak{l}$ but not to $\mathfrak{g}$. Although the open dense subset $Z \subset L / P$ is not invariant under $g_{e}$, one can show that $\Pi:=g_{e}(\Omega)$ is still a domain in $Z$. 
Assume in the following that the tripotent $e$ is maximal; i.e., $e \square e^{*}$ is invertible. Then there is a Peirce decomposition

$$
Z=X \oplus U \oplus V
$$

where

$$
X:=\left\{x \in Z:\left\{e e^{*} x\right\}=x=\left\{e x^{*} e\right\}\right\}
$$

is a "formally real" Jordan algebra with unit element $e$ and product $x \circ y:=$ $\left\{x e^{*} y\right\}$,

$$
U:=\left\{\xi \in Z:\left\{e e^{*} \xi\right\}=\xi=-\left\{e \xi^{*} e\right\}\right\}
$$

and

$$
V:=\left\{z \in Z:\left\{e e^{*} z\right\}=\frac{z}{2}\right\} .
$$

The interior of the set of squares in $X$ is a cone $\Lambda$ (cf. [BK]) and the unbounded model of the symmetric $R$-space is the Siegel domain

$$
\Pi:=\{x+\xi+v \in Z: 2 x-\Phi(v, v) \in \Lambda\} .
$$

Here we put $\Phi\left(v_{1}, v_{2}\right):=2\left\{v_{1} v_{2}^{*} e\right\}$.

In the following sections we will present a detailed (stochastic) analysis on $\Pi$, separating the three cases (i) $Z=X$, (ii) $Z=X \oplus U$, and (iii) $Z=X \oplus U \oplus V$. In case (i), the domain (2.9) is the cone $\Lambda$ itself. In case (ii), we obtain a symmetric tube domain $\Lambda \times U$. In case (iii), we obtain the (proper) Siegel domains.

In the irreducible case, the resulting symmetric spaces correspond to the root systems (cf. [L1]):

(i) $A_{r-1}$,

(ii) $C_{r}$ or $D_{r}$,

(iii) $B_{r}$ or $B C_{r}$.

It follows that our analysis covers all classical spaces and 4 exceptional spaces (of dimensions $16,26+1,32$, and 54). The remaining 13 exceptional spaces with root systems $G_{2} ; F_{4} ; E_{6} ; E_{7} ; E_{8}$ and dimensions 8,$14 ; 28,40,52,64$, $112 ; 42,78 ; 70,133 ; 128,248$ are not accessible to a purely Jordan algebraic treatment.

\section{SYMMETRIC CONES}

Every (formally real) Jordan algebra $X$ with product $x \circ y$ defines a (positivedefinite) Jordan triple product

$$
\left\{x y^{*} x\right\}=2(x \circ y) \circ x-y \circ(x \circ x)
$$

for which the unit element $e$ is a tripotent. The corresponding Cayley transform

$$
g_{e}(z)=\exp \left(\frac{\pi}{4}\left(e+z^{2}\right) \frac{\partial}{\partial z}\right)(z)=(e+z) \circ(e-z)^{-1}
$$

(cf. [L3, U1]) maps $\Omega$ onto the symmetric cone

$$
\Lambda=\left\{x^{2}: x \in X \text { invertible }\right\}
$$

associated with $X$. This cone is homogeneous under its (reductive) linear automorphism group

$$
G^{0}=G L(\Lambda):=\{P \in G L(X): P \Lambda=\Lambda\}
$$


and

$$
K^{0}=O(\Lambda):=\{P \in G L(\Lambda): P e=e\}
$$

is a maximal compact subgroup, whose elements are the Jordan algebra automorphisms of $X$.

3.5. Example. For $\mathbb{K}=\mathbb{R}, \mathbb{C}$ or $\mathbb{H}$ (quaternions), the selfadjoint $r \times r$ matrices

$$
X_{\mathbb{K}}=\left\{x \in \mathbb{K}^{r \times r}: x=x^{*}\right\}
$$

form a Jordan algebra with unit $e=1$ (identity matrix) under the commutator product

$$
x \circ y:=\frac{x y+y x}{2} .
$$

This corresponding Jordan triple product (3.1) is

$$
\left\{x y^{*} z\right\}=\frac{1}{2}\left(x y^{*} z+z y^{*} x\right)
$$

and (3.2) is the matrix cone

$$
\Lambda_{\mathbb{K}}=\left\{x=x^{*} \in \mathbb{K}^{r \times r}: x \text { positive definite }\right\} .
$$

Putting $P_{g} x:=g x g^{*}$, we have

$$
G L\left(\Lambda_{\mathbb{K}}\right)=\left\{P_{g}: g \in G L(r, \mathbb{K})(\text { invertible })\right\}
$$

and

$$
O\left(\Lambda_{\mathbb{K}}\right)=\left\{P_{g}: g \in U(r, \mathbb{K}) \text { (unitary) }\right\}
$$

For $r=3$ and $\mathbb{K}=\mathbb{O}$ (octonions), one obtains the "exceptional" cone $\Lambda_{\mathbb{O}}$ in a similar way.

3.6. Example. For $1+n \geq 3$, the Euclidean space $X=\mathbb{R}^{1, n}=\mathbb{R} \times \mathbb{R}^{n}$ of all vectors $\left(x_{0}, x_{1}, \ldots, x_{n}\right)=\left(x_{0}, x^{\prime}\right)$ has the Jordan product

$$
\left(x_{0}, x^{\prime}\right) \circ\left(y_{0}, y^{\prime}\right):=\left(x_{0} y_{0}+x^{\prime} \cdot y^{\prime}, x_{0} y^{\prime}+y_{0} x^{\prime}\right)
$$

with the unit element $e=(1,0, \ldots, 0)$. The associated symmetric cone is the forward light cone

$$
\Lambda_{1, n}:=\left\{\left(x_{0}, x_{1}, \ldots, x_{n}\right): x_{0}>\left(x_{1}^{2}+\cdots+x_{n}^{2}\right)^{1 / 2}\right\} \text {. }
$$

In this case $G L\left(\Lambda_{1, n}\right)=O(1, n)$ is the (two-component) "orthochronous" Lorentz group, and

$$
O\left(\Lambda_{1, n}\right)=\left\{\left(\begin{array}{ll}
1 & 0 \\
0 & P
\end{array}\right): P \in O(n)\right\}
$$

In order to determine the (reductive) Lie algebra $\mathfrak{g}^{0}$ associated with $\Lambda$, we apply the (adjoint) Cayley transformation and obtain for all $x \in X$ and $M \in \mathfrak{l}^{0}$ (cf. (2.4))

$$
\operatorname{Ad}\left(g_{e}\right)\left(\left(x-\left\{z x^{*} z\right\}\right) \frac{\partial}{\partial x}\right)=2\left\{x e^{*} z\right\} \frac{\partial}{\partial z}
$$

and $\operatorname{Ad}\left(g_{e}\right) M=M$. Identifying linear vector fields with the corresponding endomorphisms, it follows that $\mathfrak{g}^{0}$ has a Cartan decomposition

$$
\mathfrak{g}^{0}=\mathfrak{l}^{0} \oplus \mathfrak{p}^{0}
$$


where $\mathfrak{l}^{0}$ is the Lie algebra of (3.4) and $\mathfrak{p}^{0}$ consists of all multiplication operators

$$
M_{x} z:=x \circ z=\left\{x e^{*} z\right\}
$$

with $x \in X$. One can show that

$$
\mathfrak{g}^{0}=\mathfrak{g l}(\Lambda):=\{M \in \mathfrak{g l}(X): \exp (t m) \Lambda=\Lambda \forall t \in \mathbb{R}\}
$$

is the Lie algebra of $G L(\Lambda)$. From now on we consider only irreducible cones. Consider a complete system $e_{1}, \ldots, e_{r}$ of minimal disjoint idempotents of $X$. The cardinality $r$ of such a "frame" is an important invariant of $X$ called its rank. By [L3] there is a Peirce decomposition

$$
X=\sum_{1 \leq i \leq j \leq r} X_{i j}
$$

into mutually orthogonal "eigenspaces"

$$
X_{i j}:=\left\{x \in X: e_{k} \circ x=\frac{\delta_{i k}+\delta_{j k}}{2} x, \forall 1 \leq k \leq r\right\} .
$$

We have $X_{i i}=\mathbb{R}_{e i}(1 \leq i \leq r)$ and

$$
a:=\operatorname{dim}_{\mathbb{R}} X_{i j} \quad(1 \leq i<j \leq r)
$$

is an invariant of $X$ called the characteristic multiplicity of $X$. We put $X_{j i}:=$ $X_{i j}$. One can show that

$$
\mathfrak{a}:=\left\{\sum_{i=1}^{r} t_{i} M_{e_{i}}: t_{1}, \ldots, t_{r} \in \mathbb{R}\right\}
$$

is a maximal abelian subspace of $\mathfrak{p}^{0}$. Let

$$
M_{e_{i}}^{\#}\left(M_{e_{j}}\right):=\delta_{i j} \quad(\text { Kronecker symbol })
$$

denote the dual basis of linear forms on $a$.

3.12. Example. The matrix cones have the canonical frame

$$
e_{i}:=\left(\begin{array}{ccccccc}
0 & & & & & & \\
& \ddots & & & & & 0 \\
& & 0 & & & & \\
& & & 1 & & & \\
0 & & & & 0 & & \\
0 & & & & & & 0
\end{array}\right) \leftarrow i
$$

for $1 \leq i \leq r$, and for $i<j$ the Peirce space $X_{i j}$ consist of all selfadjoint matrices with zero coefficients except in the $(1, j)$ th place and $(j, i)$ th place. In particular $a=\operatorname{dim} X_{i j}=\operatorname{dim}_{\mathbb{R}} \mathbb{K}$.

3.13. Example. For the forward light cone $\Lambda_{1, n}$ we have $r=2$ and any pair of orthogonal vectors $e_{1}, e_{2}$ of norm $1 / \sqrt{2}$ constitutes a frame. The Peirce space $X_{12}$ is the orthogonal complement of $e_{1}$ and $e_{2}$, and $a=n-1$.

3.14. Proposition. There is a real root decomposition

$$
\mathfrak{g}^{0}=\sum_{1 \leq i, j \leq r} \mathfrak{g}_{i j}^{0} \oplus \mathfrak{m}
$$


with respect to $\mathfrak{a}$, where

$$
\mathfrak{g}_{i j}^{0}=\left\{\left\{x e_{i}^{*} z\right\} \frac{\partial}{\partial z}: x \in X_{i j}\right\}
$$

is the root space of

$$
\frac{1}{2}\left(M_{e_{j}}^{\#}-M_{e_{i}}^{\#}\right)
$$

and

$$
\mathfrak{m}:=\left\{M \in \mathfrak{l}^{0}: M e_{1}=\cdots=M e_{r}=0\right\} .
$$

Proof. As a consequence of (2.1), we have

$$
x \square y^{*}=M_{x \circ y}+\left[M_{x}, M_{y}\right] \in \mathfrak{g}^{0}
$$

for all $x, y \in X$. In particular, $\mathfrak{g}_{i j}^{0} \subset \mathfrak{g}^{0}$. Since

$$
\left[e_{i} \square e^{*}, x \square e_{i}^{*}\right]=\left\{e_{i} e^{*} x\right\} \square e_{i}^{*}-x \square e\left\{e_{i}^{*} e\right\}^{*}=-\frac{1}{2} x \square e_{i}^{*}
$$

and

$$
\left[e_{j} \square e^{*}, x \square e_{i}^{*}\right]=\left\{e_{j} e^{*} x\right\} \square e_{i}^{*}-x \square e\left\{e_{j}^{*} e\right\}^{*}=-\frac{1}{2} x \square e_{i}^{*}
$$

and $\left[e_{k} \square e^{8}, x \square e_{i}^{*}\right]=0$ whenever $k \neq i, j, \mathfrak{g}_{i j}^{0}$ is a root space for (3.16).

Now let $M \in \mathfrak{g}^{0}$ be arbitrary. Then we have $M e_{j}=\sum_{k=1}^{r} x_{j}^{k}$ with $x_{j}^{k} \in X_{j k}$, as follows from the Peirce composition rules [L3, Theorem 3.14] applied to derivations and multiplication operators, respectively. Put

$$
B:=\sum_{i}\left(x_{i}^{i}+2 \sum_{k \neq i} x_{i}^{k}\right) \square e_{i}^{*} .
$$

Then

$$
B e_{j}=x_{j}^{j}+2 \sum_{k \neq j} x_{j}^{k} / 2=M e_{j}
$$

for all $j$, showing that $M-B \in \mathfrak{m}$. Q.E.D.

\section{CONICAL AND SPHERICAL FUNCTIONS ON $\Lambda$}

Endow $\Lambda$ with the unique $G^{0}$-invariant Riemannian structure such that the basis (3.11) of $\mathfrak{a} \subset \mathfrak{p}^{0} \approx T_{e}(\Lambda)$ (tangent space) becomes orthonormal. Consider the mapping $\varphi: \mathfrak{a} \rightarrow \Lambda$ defined by

$$
\varphi\left(t_{1}, \ldots, t_{r}\right):=\exp \left(\sum_{i} t_{i} M_{e_{i}}\right)(e)=\sum_{i} e^{t_{i}} e_{i} .
$$

Let $N^{0}$ be the connected Lie subgroup with Lie algebra

$$
\mathfrak{n}^{0}:=\sum_{i<j} \mathfrak{g}_{i j}^{0}
$$

Then we have the (direct) Iwasawa decomposition [H1, FK]

$$
\Lambda=N^{0} \cdot \varphi(\mathfrak{a}) .
$$


Let $\mathscr{D}(\Lambda)$ denote the algebra of all (scalar) differential operators $D$ on $\Lambda$ which are invariant under $G L(\Lambda)$; that is, they satisfy

$$
\left(g_{*} D\right) f:=D(f \circ g) \circ g^{-1}=D f
$$

for all $f \in \mathscr{C}^{\infty}(\Lambda)$ and $g \in G L(\Lambda)$. For example the Laplace-Beltrami operator $\Delta^{0}$ of $\Lambda$ belongs to $\mathscr{D}(\Lambda)$.

By [H1, Theorem II.3.6], every $D \in \mathscr{D}(\Lambda)$ has an $N^{0}$-radial part $D_{N}$ which is a differential operator on $\mathfrak{a}$ satisfying

$$
(D f) \circ \varphi=D_{N}(f \circ \varphi)
$$

for all $N^{0}$-invariant functions $f \in \mathscr{C}^{\infty}(\Lambda)$.

4.5. Proposition. $\Delta^{0}$ has the $N^{0}$-radial part

$$
\Delta_{N}^{0}=\sum_{i}\left(\frac{\partial^{2}}{\partial t_{i}^{2}}-\frac{a}{2}(2 i-r-1) \frac{\partial}{\partial t_{i}}\right) .
$$

Proof. For any $D \in \mathscr{D}(\Lambda), D_{N}$ is a constant coefficient differential operator of the form

$$
D_{N}=q\left(\frac{\partial}{\partial t_{1}}-\rho_{1}^{0}, \ldots, \frac{\partial}{\partial t_{r}}-\rho_{r}^{0}\right)
$$

where $q$ is any symmetric (i.e., Weyl group invariant) polynomial, and

$$
\rho_{i}^{0}=\frac{a}{4}(2 i-r-1)
$$

are the coefficients of the half-sum of positive roots

$$
\rho^{0}=\frac{a}{2} \sum_{i<j} \frac{1}{2}\left(M_{e_{j}}^{\#}-M_{e_{i}}^{\#}\right)=\frac{a}{4} \sum_{i}(2 i-r-1) M_{e_{i}}^{\#}
$$

(cf. Proposition 3.14). By [H1, Proposition II.3.8], $\Delta^{0}$ corresponds to the polynomial

$$
q\left(s_{1}, \ldots, s_{r}\right):=\sum_{i}\left(s_{i}^{2}-\left(\rho_{i}^{0}\right)^{2}\right)
$$

so that

$$
\Delta_{N}^{0}=\sum_{i}\left(\frac{\partial^{2}}{\partial t_{i}^{2}}-2 \rho_{i}^{0} \frac{\partial}{\partial t_{i}}\right) \text {. Q.E.D. }
$$

The Peirce decomposition (3.9) enables us to construct $N^{0}$-invariant "conical" eigenfunctions $\Delta^{0}$ in an explicit way.

We first introduce the minors of an irreducible Jordan algebra $X$ with respect to a frame $\left\{e_{1}, \ldots, e_{r}\right\}$ of idempotents. The determinant of $X$ is the unique irreducible polynomial $N_{r}$ of degree $r$ on $X$ which satisfies the invariance property

$$
N_{r}(P x)=N_{r}(x) N_{r}(P e)
$$

for all $P \in G L(\Lambda)$ and $x \in X$ and is normalized by putting $N_{r}(e):=1$. For example, we have $N_{r}(x)=\operatorname{Det}(x)$ for selfadjoint matrices $x$ over $\mathbb{R}$ or $\mathbb{C}$, and

$$
N_{2}\left(x_{0}, \ldots, x_{n}\right)=x_{0}^{2}-x_{1}^{2}-\cdots-x_{n}^{2}
$$

in case of the light cone. 
For each $k \geq 1$, the Jordan subalgebra

$$
X_{k}:=\sum_{1 \leq i \leq j \leq k} \bigoplus X_{i j}
$$

with unit element $e_{1}+\cdots+e_{k}$ has its own determinant $N_{k}: X_{k} \rightarrow \mathbb{R}$ of degree $k$. Via the orthogonal projection $X \rightarrow X_{k}$ we may define $N_{k}$ on $X$. The polynomials $N_{1}, \ldots, N_{r}$ are called the minors of $X$. They are strictly positive on $\Lambda$.

For real or complex matrices, the $k$ th minor is given by

$$
N_{k}\left(\begin{array}{ccc}
x_{11} & \cdots & x_{1 r} \\
x_{r 1} & & x_{r r}
\end{array}\right):=\operatorname{Det}\left(\begin{array}{lll}
x_{11} & \cdots & x_{1 k} \\
x_{k 1} & & x_{k k}
\end{array}\right)
$$

whereas the light cone has

$$
N_{1}\left(x_{0}, x_{1}, \ldots, x_{n}\right)=\frac{x_{0}+x_{1}}{2}
$$

for $e_{1}:=\left(\frac{1}{2}, \frac{1}{2}, 0, \ldots, 0\right)$ and $e_{2}=\left(\frac{1}{2},-\frac{1}{2}, 0, \ldots, 0\right)$.

4.10. Proposition. For each $\alpha=\left(\alpha_{1}, \ldots, \alpha_{r}\right) \in \mathbb{C}^{r}$, the smooth function

$$
N_{\alpha}(x):=N_{1}(x)^{\alpha_{1}-\alpha_{2}} N_{2}(x)^{\alpha_{2}-\alpha_{3}} \cdots N_{r}(x)^{\alpha_{r}}
$$

on $\Lambda$ is a $N^{0}$-invariant eigenfunction of $\Delta^{0}$, with eigenvalue

$$
\chi_{\alpha}^{0}=\sum_{i=1}^{r} \alpha_{i}\left(\alpha_{i}+\frac{a}{2}(r+1-2 i)\right) .
$$

Proof. It was shown in [U2] that the minors $N_{1}, \ldots, N_{r}$ are $N^{0}$-invariant. Since

$$
\begin{aligned}
& \left(N_{\alpha} \circ \varphi\right)\left(t_{1}, \ldots, t_{r}\right)=\left(e^{t_{1}}\right)^{\alpha_{1}-\alpha_{2}}\left(e^{t_{1}+t_{2}}\right)^{\alpha_{2}-\alpha_{3}} \cdots\left(e^{t_{1}+\cdots+t_{r}}\right)^{\alpha_{r}} \\
& =e^{t_{1} \alpha_{1}+t_{2} \alpha_{2}+\cdots+t_{r} \alpha_{r}},
\end{aligned}
$$

every $D \in \mathscr{D}(\Lambda)$ with radial part (4.6) satisfies

$$
D N_{\alpha}=q\left(\alpha_{1}-\rho_{1}^{0}, \ldots, \alpha_{r}-\rho_{r}^{0}\right) N_{\alpha} .
$$

For the polynomial (4.7), we obtain

$$
\Delta^{0} N_{\alpha}=\sum_{i}\left(\alpha_{i}^{2}-2 \alpha_{i} \rho_{i}^{0}\right) N_{\alpha} \text {. Q.E.D. }
$$

The functions (4.9) are called conical functions on $\Lambda$. Put

$$
\overrightarrow{\mathbb{R}}^{r}:=\left\{\alpha \in \mathbb{R}^{r}: \alpha_{1} \geq \alpha_{2} \geq \cdots \geq \alpha_{r}\right\}
$$

and

$$
\overrightarrow{\mathbb{N}}^{r}:=\overrightarrow{\mathbb{R}}^{r} \cap \mathbb{N}^{r}
$$

The elements $\alpha=\left(\alpha_{1}, \ldots, \alpha_{r}\right) \in \overrightarrow{\mathbb{N}}^{r}$ are called partitions of $|\alpha|:=\alpha_{1}+\cdots+\alpha_{r}$. For each $\alpha \in \overrightarrow{\mathbb{N}}^{r}, N_{\alpha}$ is a polynomial of degree $|\alpha|$ on $X$ called the conical polynomial of type $\alpha$.

We now pass to the maximal compact subgroup $K^{0}=O(\Lambda)$ of $G L(\Lambda)$. Let

$$
\mathfrak{a}_{+}^{0}:=\left\{\sum_{i=1}^{r} t_{i} M_{e_{i}}: t_{1}<\cdots<t_{r}\right\}
$$


denote the "Weyl chamber". Then there is a Cartan decomposition [H1]

$$
\Lambda=K^{0} \cdot \varphi\left(\mathfrak{a}_{+}^{0}\right) \text {. }
$$

By [H1, Theorem II.3.6], every $D \in \mathscr{D}(\Lambda)$ has a $K^{0}$-radial part $D_{K}$ which is a differential operator on $\mathfrak{a}_{+}^{0}$ satisfying

$$
(D f) \circ \varphi=D_{K}(f \circ \varphi)
$$

for all $K^{0}$-invariant functions $f \in \mathscr{C}^{\infty}(\Lambda)$.

4.16. Proposition. $\Delta^{0}$ has the $K^{0}$-radial part

$$
\Delta_{K}^{0}=\sum_{i=1}^{r}\left(\frac{\partial^{2}}{\partial t_{i}^{t}}-\frac{a}{2}(r-1) \frac{\partial}{\partial t_{i}}\right)+a \sum_{i \neq j} \frac{1}{\exp \left(t_{j}-t_{i}\right)-1} \frac{\partial}{\partial t_{i}} .
$$

Proof. The positive roots (3.16) induce the "density function"

$$
\delta\left(t_{1}, \ldots, t_{r}\right):=\prod_{i<j} \sinh ^{a}\left(\frac{t_{j}-t_{i}}{2}\right)=\prod_{i<j}\left(\frac{1}{2}\left(e^{t_{j}}-e^{t_{i}}\right) e^{-\left(t_{i}+t_{j}\right) / 2}\right)^{a}=\operatorname{const} \cdot \omega^{a} \pi
$$

where

$$
\omega=\prod_{i<j}\left(e^{t_{j}}-e^{t_{i}}\right), \quad \pi=\left(\prod_{i} e^{t_{i}}\right)^{-a(r-1) / 2} .
$$

By $[H 1$, p. 335] we have

$$
\begin{aligned}
\Delta_{K}^{0} & =\frac{1}{\delta} \sum_{i} \frac{\partial}{\partial t_{i}} \delta \frac{\partial}{\partial t_{i}}=\frac{1}{\omega^{a} \pi} \sum_{i} \frac{\partial}{\partial t_{i}} \omega^{a} \pi \frac{\partial}{\partial t_{i}} \\
& =\sum_{i}\left(\frac{\partial}{\partial t_{i}}+\frac{1}{\pi} \frac{\partial \pi}{\partial t_{i}}+\frac{a}{\omega} \frac{\partial}{\partial t_{i}}\right) \frac{\partial}{\partial t_{i}} \\
& =\sum_{i}\left(\frac{\partial}{\partial t_{i}}-\frac{a}{2}(r-1)+a \sum_{j \neq i} \frac{1}{\exp \left(t_{j}-t_{i}\right)-1}\right) \frac{\partial}{\partial t_{i}} \text {. Q.E.D. }
\end{aligned}
$$

\subsection{Corollary.}

$$
\begin{aligned}
\varphi_{*} \Delta_{K}^{0}= & \sum_{i}\left(x_{i}^{2} \frac{\partial^{2}}{\partial x_{i}^{2}}+\left(1-\frac{a}{2}(r-1)\right) x_{i} \frac{\partial}{\partial x_{i}}\right) \\
& +a \sum_{i \neq j} \frac{x_{i}^{2}}{x_{j}-x_{i}} \frac{\partial}{\partial x_{i}} .
\end{aligned}
$$

Proof. Putting $x_{i}=e^{t_{i}}$ we have by (4.18)

$$
\omega=\prod_{i<j}\left(x_{j}-x_{i}\right), \quad \pi=\left(x_{1} \cdots x_{r}\right)^{-a(r-1) / 2}
$$

and (4.17) implies

$$
\begin{aligned}
\varphi_{*} \Delta_{K}^{0} & =\sum_{i}\left(\frac{d^{2} x_{i}}{d t_{i}^{2}}+\left(\frac{d x_{i}}{d t_{i}}\right)^{2}\left(\frac{\partial}{\partial x_{i}}+\frac{1}{\pi} \frac{\partial \pi}{\partial x_{i}}+\frac{a}{\omega} \frac{\partial \omega}{\partial x_{i}}\right)\right) \frac{\partial}{\partial x_{i}} \\
& =\sum_{i}\left(x_{i}+x_{i}^{2}\left(\frac{\partial}{\partial x_{i}}-\frac{a}{2}(r-1) \frac{1}{x_{i}}+a \sum_{j \neq i} \frac{1}{x_{j}-x_{i}}\right)\right) \frac{\partial}{\partial x_{i}} \text {. Q.E.D. }
\end{aligned}
$$


By integrating over the compact Lie group $K^{0}$ we obtain a smooth $K^{0}$-invariant function

$$
K_{\alpha}^{0}(x):=\int_{K^{0}} N_{\alpha}(s x) d s
$$

on $\Lambda$, called the spherical function of type $\alpha \in \mathbb{C}^{r}$. In case $\alpha \in \overrightarrow{\mathbb{N}}^{r}, K_{\alpha}^{0}$ is a polynomial on $X$ called the spherical polynomial of type $\alpha$. Applying Proposition 4.9, we obtain

$$
\Delta^{0} K_{\alpha}^{0}=\chi_{\alpha}^{0} K_{\alpha}^{0}
$$

for every $\alpha \in \mathbb{C}^{r}$.

4.21. Proposition. For $\alpha \in \overrightarrow{\mathbb{N}}^{r}$, the spherical polynomial $K_{\alpha}^{0}$ has a finite expansion

$$
K_{\alpha}^{0}\left(\sum_{i=1}^{r} x_{i} e_{i}\right)=\sum_{\beta \in \mathbb{N}^{r}} c_{\beta} q_{\beta}\left(x_{1}, \ldots, x_{r}\right)
$$

where $c_{\beta} \geq 0$ and $q_{\beta}$ is the monomial symmetric function. Proof. Given $s \in K^{0}$ and $l \leq r$, consider the expansion

$$
N_{l}\left(s \cdot \sum_{i=1}^{r} x_{i} e_{i}\right)=\sum_{\mu} b_{\mu}(s) x_{1}^{\mu_{1}} \cdots x_{r}^{\mu_{r}}
$$

where $\mu \in \mathbb{N}^{r}$ (not necessarily nonincreasing). Define

$$
|\mu|:=\mu_{1}+\cdots+\mu_{r}
$$

and

$$
l(\mu):=\operatorname{card}\left\{i: \mu_{i} \neq 0\right\} .
$$

If $|\mu| \neq l$, then $b_{\mu}(s)=0$ since $N_{l}$ is homogeneous of degree $l$. Thus we may assume $|\mu|=l$ and therefore $l(\mu) \leq l$. Now assume $l(\mu)<l$. Put $I:=\left\{i: \mu_{i}>0\right\}$. Then for every choice of $x_{i}(i \in I)$ we have

$$
N_{l}\left(s \cdot \sum_{i \in I} x_{i} e_{i}\right)=N_{X_{l}}\left(P_{l}\left(s \cdot \sum_{i \in I} x_{i} e_{i}\right)\right)=0
$$

since

$$
\begin{gathered}
\operatorname{rank} P_{l}\left(s \cdot \sum_{i \in I} x_{i} e_{i}\right) \leq \operatorname{rank} s \cdot \sum_{i \in I} x_{i} e_{i} \\
=\operatorname{rank} \sum_{i \in I} x_{i} e_{i}=\operatorname{card}(I)<l .
\end{gathered}
$$

Here $P_{l}: X \rightarrow X_{l}$ is the orthogonal projection (which may decrease the rank) and $N_{X_{l}}$ is the determinant of the Jordan algebra $X_{l}$ of rank $l$, defined in (4.9). It follows that $b_{\mu}(s)=0$ whenever $l(\mu)<l$. Thus (4.22) simplifies to

$$
N_{l}\left(s \cdot \sum_{i=1}^{r} x_{i} e_{i}\right)=\sum_{I} b_{I}(s) \prod_{i \in I} x_{i}
$$


where $I$ runs over all subsets of $\{1, \ldots, r\}$ having $l$ elements. Since $N_{l} \geq 0$ on the closure $\bar{\Lambda}$, we have

$$
b_{I}(s)=N_{l}\left(s \cdot \sum_{i \in I} e_{i}\right) \geq 0
$$

for every $s \in K^{0}$. Taking powers and products, (4.23) implies

$$
N_{\alpha}\left(s \cdot \sum_{i=1}^{r} x_{i} e_{i}\right)=\sum_{\substack{\mu \in \mathbb{N}^{r} \\|\mu|=|\alpha|}} b_{\mu}(s) x_{1}^{\mu_{1}} \cdots x_{r}^{\mu_{r}}
$$

where $b_{\mu}(s) \geq 0$ for all $s \in K^{0}$. Applying (4.20), this implies

$$
K_{\alpha}^{0}\left(\sum_{i=1}^{r} x_{i} e_{i}\right)=\sum_{\substack{\mu \in \mathbb{N}^{r} \\|\mu|=|\alpha|}} b_{\mu}(s) x_{1}^{\mu_{1}} \cdots x_{r}^{\mu_{r}}
$$

where

$$
b_{\mu}=\int_{K^{0}} b_{\mu}(s) d s \geq 0 .
$$

For every permutation $\sigma \in \mathfrak{S}_{r}$ there exists an element $s \in K^{0}$ such that

$$
s e_{i}=e_{\sigma(i)} \quad(1 \leq i \leq r) .
$$

Putting $\sigma \cdot \mu:=\left(\mu_{\sigma(1)}, \ldots, \mu_{\sigma(r)},(4.24)\right.$ implies $b_{\sigma \cdot \mu}=b_{\mu}$ since $K_{\alpha}^{0}$ is $K^{0}$ invariant. Since

$$
q_{\beta}\left(x_{1}, \ldots, x_{r}\right)=\sum_{\mu} x_{1}^{\mu_{1}} \cdots x_{r}^{\mu_{r}}
$$

where $\mu$ runs over all distinct permutations of $\beta$, the assertion follows. Q.E.D.

\section{STOCHASTIC CONTROL THEORY ON SYMMETRIC CONES: CONICAL FUNCTIONS}

To commence the study of stochastic control problems in noncompact symmetric spaces of arbitrary rank, a symmetric cone is considered where the cost functional is $N^{0}$-invariant. Since the $N^{0}$-radial part of the Laplace-Beltrami operator is a constant coefficient differential operator and the controlled diffusion can be projected to a Euclidean space, the analysis of the control problem is quite straightforward. However this problem serves to introduce some of the notions in an elementary context.

A stochastic control problem is formulated in a symmetric cone using the $N^{0}$-invariance and a conical polynomial $N_{\alpha}$. The stochastic control problem is the control of a Brownian motion in a symmetric cone $\Lambda$ so that the controlled diffusion remains close to the base point of $\Lambda$. Using the $N^{0}$-invariance of the cost functional to be introduced and the Iwasawa decomposition of the Lie group we can use the coordinates $t=\left(t_{1}, \ldots, t_{r}\right)$ for the Cartan subalgebra, $\mathfrak{a}$.

The controlled diffusion in the symmetric cone has the infinitesimal generator

$$
\frac{1}{2} \Delta^{0}+\left\langle u(s, t), \nabla_{t}\right\rangle \text {. }
$$


Since the $N^{0}$-invariance of the control problem is exploited, it suffices to consider the controlled diffusion projected to the abelian subgroup or equivalently the Cartan subalgebra $\mathfrak{a}$. The infinitesimal generator for the controlled diffusion projected to $\mathfrak{a}$ is

$$
\frac{1}{2} \Delta_{N}^{0}+\left\langle u(s, t), \nabla_{t}\right\rangle
$$

where $\Delta_{N}^{0}$ is the $N^{0}$-radial part of the Laplace-Beltrami operator $\Delta^{0}, t=$ $\left(t_{1}, \ldots, t_{r}\right), \nabla_{t}=\left(\frac{\partial}{\partial t_{1}}, \ldots, \frac{\partial}{\partial t_{r}}\right)$ is the gradient, and $s \in \mathbb{R}_{+}$. Suppose that $(X(s), s \in[0, T])$ is the controlled diffusion in $\mathfrak{a}$ with infinitesimal generator (5.1) where the dependence of $X$ on $u$ has been suppressed. The cost functional for the stochastic control problem is

$$
J_{N}^{0}(U, \alpha)=E_{X(0)} \int_{0}^{T}\left(N_{\alpha} \circ \varphi\right)(X(s))+\langle U(s), U(s)\rangle d s
$$

where $N_{\alpha}$ is the $N^{0}$-invariant polynomial eigenfunction of $\Delta^{0}$ for the partition $\alpha$.

Since the infinitesimal generator of $(X(s), s \in[0, T])$ is $(5.1)$, the family of stochastic differential equations that $(X(s), s \in[0, T])$ satisfies is

$$
d X_{k}(s)=\left[-\frac{a}{4}(2 k-r-1)+U_{k}(s, X(s))\right] d s+d B_{k}(s)
$$

for $k=1,2, \ldots, r, X(0) \in \mathfrak{a}$, and $\left(B_{1}(s), \ldots, B_{r}(s), s \geq 0\right)$ is a standard $r$-dimensional Brownian motion.

To describe the family of admissible controls it suffices to give the requirements in terms of $(X(s), s \in[0, T])$ by the $N^{0}$-invariance of (5.1). An admissible control at time $s$ is a Borel measurable function of $X(s)$ so that the family of stochastic differential equations (5.3) has one and only one strong solution.

It is elementary to verify that the stochastic control problem $(5.1,5.2)$ is well posed; that is, there is at least one control law (e.g. $U \equiv 0$ ) that gives a finite value to (5.2).

The solution of the stochastic control problem is given now.

5.4. Theorem. The stochastic control problem described by $(5.1,5.2)$ has an optimal control given by

$$
U^{*}(s, t)=-\frac{g(s)}{2} \nabla_{t}\left(N_{\alpha} \circ \varphi\right)(t)
$$

where $s \in[0, T], t=\left(t_{1}, \ldots, t_{r}\right), \nabla_{t}$ is the t-gradient, and $g$ is the unique positive solution of the scalar Riccati differential equation

$$
\frac{d g}{d s}+\frac{1}{2} \chi_{\alpha}^{0} g-\frac{|\alpha|}{4} g^{2}+1=0, \quad g(T)=0,
$$

where

$$
\chi_{\alpha}^{0}=\sum_{i=1}^{r}\left(\alpha_{i}^{2}-2 \alpha_{i} \rho_{i}^{0}\right) \quad \text { and } \rho_{i}^{0}=\frac{a}{4}(2 i-r-1) .
$$

Proof. A verification theorem [FR, p. 134] is applied to the smooth solution of the Hamilton-Jacobi equation

$$
0=\frac{\partial W}{\partial s}+\min _{v \in \mathbb{R}^{r}}\left[\frac{1}{2} \Delta_{N}^{0} W+\sum_{j=1}^{r} v_{j} \frac{\partial W}{\partial t_{j}}+\left(N_{\alpha} \circ \varphi\right)(t)+\sum_{j=1}^{r} v_{j}^{2}\right] .
$$


The family of controls obtained by the minimization in (5.6) is

$$
U_{j}^{*}(s, t)=-\frac{1}{2} \frac{\partial W(s, t)}{\partial t_{j}}
$$

for $j=1,2, \ldots, r$.

Assume a solution $W$ of $(5.7)$ as

$$
W(s, t)=g(s)\left(N_{\alpha} \circ \varphi\right)(t) .
$$

Substituting (5.9) into (5.7) it follows that

$$
\begin{aligned}
0 & =g^{\prime} N_{\alpha} \circ \varphi+\frac{1}{2} \chi_{\alpha}^{0} N_{\alpha} \circ \varphi-\frac{|\alpha|}{4} g^{2} N_{\alpha} \circ \varphi+N_{\alpha} \circ \varphi \\
& =N_{\alpha} \circ \varphi\left(g^{\prime}+\frac{1}{2} \chi_{\alpha}^{0} g-\frac{|\alpha|}{4} g^{2}+1\right) .
\end{aligned}
$$

If $g$ in (5.10) satisfies (5.6) then (5.9) is a smooth solution of (5.7) that satisfies the boundary condition $W(s, t)=0$ for $(s, t) \in\{T\} \times \mathfrak{a}$. The family of controls (5.5) is an admissible (optimal) control if the family of stochastic differential equations (5.3) with these controls has one and only one strong solution on $[0, T]$.

Since the coefficients of the family of stochastic differential equations (5.3) with the controls $(5.8)$ are locally smooth in $\mathfrak{a}$, a unique strong solution can be constructed at least locally. Thus it suffices to verify that there is no escape of the process in $[0, T]$. Define the random times

$$
S_{i}=\left\{\begin{array}{l}
\inf \left\{s \in[0, T]: X_{i}(s)=+\infty\right\} \\
+\infty \text { if the above set is empty }
\end{array}\right.
$$

for $i=1,2, \ldots, r$. It is easy to verify by contradiction that

$$
P\left(S_{i}<\infty \text { for some } i=1,2, \ldots, r\right)=0
$$

by comparing (5.3) to Brownian motion with no drift term. Thus (5.5) is an admissible optimal control. Q.E.D.

\section{STOCHASTIC CONTROL THEORY ON SYMMETRIC CONES: \\ SPHERICAL FUNCTIONS}

A stochastic control problem is described in a symmetric cone using a $K^{0}$ invariance and a spherical polynomial $K_{\alpha}^{0}$. The stochastic control problem is the control of a Brownian motion in a symmetric cone $\Lambda$ by a drift vector field so that this controlled diffusion remains close to the base point of $\Lambda$.

The controlled diffusion in the symmetric cone $\Lambda$ has the infinitesimal generator

$$
\frac{1}{2} \Delta^{0}+\left\langle u(s, t), \nabla_{t}\right\rangle
$$

where $\Delta^{0}$ is the Laplace-Beltrami operator on $\Lambda, t=\left(t_{1}, \ldots, t_{r}\right)$ is the coordinator in $\mathfrak{a}_{+}^{0}$, and $s \in \mathbb{R}_{+}$. Since the $K^{0}$-invariance of the control problem is exploited, it suffices to consider the controlled diffusion projected to the positive Weyl chamber $\mathfrak{a}_{+}^{0}$ with the infinitesimal generator

$$
\frac{1}{2} \Delta_{K}^{0}+\left\langle u(s, t), \nabla_{t}\right\rangle .
$$


Let $(X(s), s \geq 0)$ be the controlled diffusion in the positive Weyl chamber whose infinitesimal generator is (6.2). The dependence on $u$ has been suppressed. This controlled diffusion is the projection to the positive Weyl chamber by a suitable $K^{0}$-invariance. The cost functional for the stochastic optimal control problem is

$$
J_{K}^{0}(U, \alpha)=E_{X(0)} \int_{0}^{T}\left(K_{\alpha}^{0} \circ \varphi\right)(X(s))+\left\langle A^{0}(X(s)) U(s, X(s)), U(s, X(s))\right\rangle d s
$$

where $K_{\alpha}^{0}$ is the spherical polynomial (4.24) for the partition $\alpha, A^{0}(t):=$ $\operatorname{diag}\left(f_{1}(t), \ldots, f_{r}(t)\right)$,

$$
f_{i}(t)=\frac{1}{{ }_{i} K_{\alpha}^{0}(\varphi(t))}\left[\frac{\partial}{\partial t_{i}}\left(K_{\alpha}^{0} \circ \varphi\right)(t)\right]^{2},
$$

and

for $i=1,2, \ldots, r$. It is clear that

$$
{ }_{i} K_{\alpha}^{0}(x)=\frac{x_{i}}{|\alpha|} \frac{\partial}{\partial x_{i}} K_{\alpha}^{0}(x)
$$

$$
\sum_{i=1}^{r}{ }_{i} K_{\alpha}^{0}(x)=K_{\alpha}^{0}(x) .
$$

Since the infinitesimal generator of $(X(s), s \in[0, T])$ is $(6.2)$, the family of stochastic differential equations that $(X(s), s \in[0, T])$ satisfies is

$$
\begin{aligned}
& d X_{k}(s)=\left[-\frac{a}{4}(r-1)+\frac{a}{2} \sum_{j \neq k} \frac{1}{1-\exp (} X_{j}(s)-X_{k}(s)\right) \\
&\left.+U_{k}(s, X(s))\right] d s+d B_{k}(s)
\end{aligned}
$$

for $k=1,2, \ldots, r, X(0)=\left(X_{1}(0), \ldots, X_{r}(0)\right), X_{1}(0)>X_{2}(0)>\cdots>$ $X_{r}(0), X(s) \in \mathfrak{a}_{+}^{0}$, and $\left(B_{1}(s), \ldots, B_{r}(s) ; s \geq 0\right)$ is a standard $r$-dimensional Brownian motion.

Let $(Z(s) \in \Lambda, s \in[0, T])$ be the controlled diffusion with infinitesimal generator $(6.1)$ where the dependence on the control law $U(\cdot)$ has been suppressed for notational simplicity. An admissible control at time $s$ is a Borel measurable function of $Z(s)$ such that the stochastic differential equation whose generator is (6.1) has one and only one strong solution. By the $K^{0}$-invariance of the cost functional it suffices to consider controls at time $s$ that are Borel measurable functions of $X(s)$ that satisfy (6.4) so that this family of stochastic differential equations has one and only one strong solution.

Initially it is necessary to verify that the stochastic control problem $(6.2,6.3)$ is well posed, specifically that there is at least one control law that gives a finite value to $J_{\alpha}^{0}(U)$ in $(6.3)$.

6.5. Lemma. Let $(\widetilde{X}(s), s \in[0, T])$ be the diffusion process in the positive Weyl chamber with the infinitesimal generator $\frac{1}{2} \Delta_{K}^{0}$, that is, the unique strong solution of (6.4) with $U(t) \equiv 0$. Then

$$
E_{X(0)} \int_{0}^{T}\left(K_{\alpha}^{0} \circ \varphi\right)(\widetilde{X}(s)) d x<\infty .
$$


Proof. The existence and the uniqueness of a strong solution of (6.4) with $U(s) \equiv 0$ follows immediately when it is verified that $(\widetilde{X}(s), s \in[0, T])$ does not hit the walls of the positive Weyl chamber. Since this verification follows by using a comparison theorem for stochastic differential equations [IW, p. 352] in a simpler manner than in the proof of Theorem 9.7, it is omitted here.

Let $R$ be the Riemann curvature tensor of $\Lambda$. The Ricci tensor field $S$ is defined as

$$
S(X, Y)=\sum_{i=1}^{n} R\left(V_{i}, Y, V_{i}, X\right)
$$

where $X, Y \in T_{X} \Lambda, \operatorname{dim} \Lambda=n$, and $\left(V_{1}, \ldots, V_{n}\right)$ is an orthonormal basis of $T_{x} M . S(\cdot, \cdot)$ is symmetric. Let $\rho$ be defined as $\rho(X)=S(X, X)$ where $|X|=1$. Since $\Lambda$ is a Riemannian homogeneous space, it suffices to compute the Riemannian curvature and the Ricci curvature at one point. It follows that there is a $b<0$ such that $(n-1) b \leq \rho(X)$ for a unit vector $X \in T_{x} \Lambda$ and $x \in \Lambda$. Define the real-valued process $(R(s), s \in[0, T])$ as

$$
R(s)=d(e, \tilde{X}(s))
$$

where $e$ is the base point of $\Lambda, d(\cdot, \cdot)$ is the distance function on $\Lambda$, and $(\widetilde{X}(s), s \in[0, T])$ is the process given in the statement of the lemma. Let $\left(Y_{b}(s), s \in[0, T]\right)$ be the standard Brownian motion in the real hyperbolic space $\mathbb{H}^{n}(\mathbb{R})$ with the negative sectional curvature $b$. It follows [IW, p. 380] that

$$
P\left(Y_{b}(s) \geq R(s) ; s \in[0, T]\right)=1 .
$$

Thus is suffices to verify

$$
E_{X(0)} \int_{0}^{T}\left(K_{\alpha}^{0} \circ \varphi\right)\left(Y_{b}(s)\right) d s<\infty .
$$

This verification is easily made [D5, Lemma]. Q.E.D.

The solution of the stochastic control problem $(6.1,6.3)$ is given now.

6.7. Theorem. The stochastic control problem described by $(6.1,6.3)$ has an optimal control $U^{*}$ given by

$$
U^{*}(s, t)=-\frac{g(s)}{2} A^{-1}(t) \nabla_{t}\left(K_{\alpha}^{0} \circ \varphi\right)(t)
$$

where $s \in[0, T], t=\left(t_{1}, \ldots, t_{r}\right), \nabla_{t}$ is the t-gradient, and $g$ is the unique positive solution of the scalar Riccati differential equation

$$
\frac{d g}{d s}+\frac{1}{2} \chi_{\alpha}^{0} g-\frac{1}{4} g^{2}+1=0, \quad g(T)=0,
$$

where $\chi_{\alpha}^{0}$ is the eigenvalue (4.12).

Proof. It is well known (e.g. [FR]) that the Hamilton-Jacobi or dynamic programming equation for a stochastic optimal control problem of diffusion type is

$$
0=\frac{\partial W}{\partial s}+\min _{u \in U}\left[A^{v}(s) W+L(s, x, v)\right]
$$

where $A^{v}$ is the infinitesimal generator of the diffusion using the control $v$ and $L$ is the integrand of the cost functional. To apply a verification theorem [FR, 
p. 139] to the problem here it is required that the solution $W$ of $(6.10)$ with the boundary condition $W(s, y)$ for $(s, y) \in\{T\} \times \Lambda$ is $\mathscr{C}^{1,2}[(0, T) \times \Lambda]$ and continuous on $[0, T] \times \Lambda$.

To apply the Hamilton-Jacobi equation to $(6.1,6.3)$ it is important to use the fact that the integrand of the cost functional as a function of the state is $K^{0}$-invariant so that the Hamilton-Jacobi equation (6.10) can be reduced to the radial part of $(6.10)$ as

$$
0=\frac{\partial W}{\partial s}+\min _{v \in \mathbb{R}^{r}}\left[\frac{1}{2} \Delta_{K}^{0} W+\sum_{j=1}^{r} v_{j} \frac{\partial W}{\partial t_{j}}+\left(K_{\alpha}^{0} \circ \varphi\right)(t)+\sum_{j=1}^{r} f_{j} v_{j}^{2}\right]
$$

It is clear that the family of controls obtained by the minimization in (6.11) is

$$
U_{j}^{*}(s, t)=-\frac{1}{2 f_{j}(t)} \frac{\partial W(s, t)}{\partial t_{j}}
$$

for $j=1,2, \ldots, r$.

Assume a solution $W$ of $(6.11)$ as

$$
W(s, t)=\left(K_{\alpha}^{0} \circ \varphi\right)(t) g(s) .
$$

Substituting (6.13) into (6.11) it follows that

$$
\begin{aligned}
0 & =g^{\prime} K_{\alpha}^{0} \circ \varphi+\frac{1}{2} \chi_{\alpha}^{0} g K_{\alpha}^{0} \circ \varphi-\frac{1}{4} g^{2} K_{\alpha}^{0} \circ \varphi+K_{\alpha}^{0} \circ \varphi \\
& =K_{\alpha}^{0} \circ \varphi^{\prime}\left(g^{\prime}+\frac{1}{2} \chi_{\alpha}^{0} g-\frac{1}{4} g^{2}+1\right) .
\end{aligned}
$$

If $g$ in (6.14) satisfies (6.9) then (6.13) is a smooth solution of (6.11) that satisfies the boundary condition $W(s, t)=0$ for $(s, t) \in\{T\} \times \mathfrak{a}_{+}^{0}$. The family of controls (6.12) is an admissible (optimal) control if the family of stochastic differential equations (6.4) with these controls has one and only one strong solution in $[0, T]$.

Since the coefficients of the family of stochastic differential equations (6.4) with the controls $(6.12)$ are locally in $\mathfrak{a}_{+}^{0}$, a unique strong solution can be constructed at least locally.

Let $(X(s), s \in[0, T])$ be the solution of the family of stochastic differential equations (6.4) with the family of controls (6.12). There is no finite escape time by the methods in the proof of Lemma 6.5. If it is assumed that $(X(s), s \in[0, T])$ does not hit the walls of the positive Weyl chamber then the local smoothness of the coefficients of the stochastic differential equations for $(X(s), s \in[0, T])$ can be used to construct a unique strong global solution (e.g. [IW, p. 164]).

Now it is verified that $(X(s), s \in[0, T])$ does not hit the walls of the Weyl chamber. It suffices to consider one wall of the Weyl chamber because the intersection of the walls of the Weyl chamber can be treated in a similar fashion.

Choose two positive constants $K \gg c>0$ and define $\Gamma_{k}(c, K) \subset \mathfrak{a}_{+}^{0}$ for $k=\{1, \ldots, r-1\}$ as

$$
\begin{aligned}
\Gamma_{k}(c, K)=\left\{t: K>t_{1}-r c>\cdots>t_{k+2}-(r-k+1) c>t_{k+1}\right. \\
\left.\geq t_{k}>t_{k-1}-k c>\cdots>t_{r-1}-2 c>t_{r}>-K\right\} .
\end{aligned}
$$


To investigate the process $(X(s), s \in[0, T])$ in a neighborhood of $\left\{t: t_{k}=t_{k+1}\right\} \cap \Gamma_{k}(c, K)$ it suffices to consider the stochastic differential equation for

$$
Y_{k}(s)=X_{k}(s)-X_{k+1}(s)
$$

for $s \in[0, T]$ and show that $\left(Y_{k}(s), s \in[0, T]\right)$ does not hit zero. The stochastic differential equation for $\left(Y_{k}(s), s \in[0, T]\right)$ is

$$
\begin{aligned}
d Y_{k}(s)=\left[\frac{a}{2} \sum_{j \neq k}\right. & \frac{1}{1-\exp \left(X_{j}(s)-X_{k}(s)\right)} \\
& -\frac{a}{2} \sum_{j \neq k+1} \frac{1}{1-\exp \left(X_{j}(s)-X_{k+1}(s)\right)} \\
& \left.\quad+U_{k}(s, X(s))-U_{k+1}(s, X(s))\right] d s+d B_{k}(s)-d B_{k+1}(s) .
\end{aligned}
$$

It is assumed that $(X(s), s \in[0, T])$ is localized to $\Gamma_{k}(c, K)$.

Let $(Z(s), s \in[0, T])$ be the so-called two-dimensional Bessel process that is the unique strong solution of the stochastic differential equation

$$
d Z(s)=\frac{1}{Z(s)} d s+\sqrt{2} d B(s)
$$

where $(B(s), s \geq 0)$ is a real-valued standard Brownian motion.

It is convenient to regroup the drift terms in (6.17) as

$$
\begin{aligned}
d Y_{k}(s)= & {\left[\frac{a / 2}{1-\exp \left(X_{k+1}(s)-X_{k}(s)\right)}\right.} \\
& +\sqrt{2} d B(s)
\end{aligned}
$$

where

$$
\sup _{(s, x) \in[0, T] \times \Gamma_{k}(c, K)}|h(s, x)|<M<\infty .
$$

By the expansion of the exponential function there is a $\delta>0$ such that for $x \in(0, \delta]$

$$
-M+\frac{1 / 2}{1-e^{-x}}-\frac{1 / 2}{1-e^{x}}>\frac{1}{x} .
$$

Localizing $\{X(s), s \in[0, T]\}$ to $\left\{t: t_{k}-t_{k+1}<\delta\right\} \cap \Gamma_{k}(c, K)$ the stochastic differential equations (6.4) and (6.17) can be compared [IW, p. 352].

Choosing sequences $c_{n} \downarrow 0$ and $K_{n} \uparrow+\infty$ and applying the above construction to $\Gamma_{n}\left(c_{n}, K_{n}\right)$ it follows that

$$
\begin{array}{r}
P\left(X_{1}(s)>X_{2}(s)>\cdots>X_{k}(s)=X_{k+1}(s)>\cdots>X_{r}(s)\right. \\
\text { for some } s \in[0, T])=0 .
\end{array}
$$

This completes the proof of the theorem. Q.E.D. 


\section{SYMMETRIC TUBE AND SIEGEL DOMAINS}

Every Jordan *-algebra $Z$ with product $z \circ w$, unit element $e$, and involution $z^{*}$ defines a Jordan triple product

$$
\left\{z w^{*} z\right\}=2\left(z \circ w^{*}\right) \circ z-w^{*} \circ(z \circ z)
$$

for which $e$ is a tripotent. The corresponding Cayley transform

$$
g_{e}(z)=\exp \left(\frac{\pi}{4}\left(e+z^{2}\right) \frac{\partial}{\partial z}\right)(z)=(e+z) \circ(e-z)^{-1}
$$

maps $\Omega$ onto the symmetric tube domain

$$
\Pi=\left\{z \in Z: z+z^{*} \in \Lambda\right\}=\Lambda \times U,
$$

where $\Lambda$ is the symmetric cone of the formally real Jordan algebra

$$
X:=\left\{x \in Z: x^{*}=x\right\},
$$

and

$$
U:=\left\{\xi \in Z: \xi^{*}=-\xi\right\}
$$

Of particular importance is the case $U=i X$; i.e., $Z$ is the complexification of a formally real Jordan algebra $X$ endowed with the complexified Jordan product and the involution

$$
(x+i y)^{*}:=x-i y \quad(x, y \in X) .
$$

In this case $\Pi=\Lambda \times i X$ is a complex hermitian symmetric space. A basic example is Siegel's (right) half-space, corresponding to the cone $\Lambda_{\mathbb{R}}$ of positive definite symmetric matrices (cf. Example 3.5). In order to determine the (semisimple) Lie algebra $\mathfrak{g}$ associated with $\Pi$, we apply the (adjoint) Cayley transformation and obtain for all $x \in X, \xi \in U$, and $M \in \mathfrak{l}^{0}$ (cf. (3.7)).

$$
\begin{gathered}
\operatorname{Ad}\left(g_{e}\right)\left(\left(x-\left\{z x^{*} z\right\}\right) \frac{\partial}{\partial z}\right)=2\left\{x e^{*} z\right\} \frac{\partial}{\partial z}, \\
\operatorname{Ad}\left(g_{e}\right)\left(\left(\xi-\left\{z \xi^{*} z\right\}-2\left\{\xi e^{*} z\right\}\right) \frac{\partial}{\partial z}\right)=2 \xi \frac{\partial}{\partial z}, \\
\operatorname{Ad}\left(g_{e}\right)\left(\left(\xi-\left\{z \xi^{*} z\right\}+2\left\{\xi e^{*} z\right\}\right) \frac{\partial}{\partial z}\right)=2\left\{z \xi^{*} z\right\} \frac{\partial}{\partial z}
\end{gathered}
$$

and

$$
\operatorname{Ad}\left(g_{e}\right) M=M .
$$

It follows that $\mathfrak{g}$ has a Cartan decomposition

$$
\mathfrak{g}=\mathfrak{l} \oplus \mathfrak{p},
$$

where

$$
\mathfrak{l}=\mathfrak{l}^{0} \oplus\left\{\left(\xi+\left\{z \xi^{*} z\right\}\right) \frac{\partial}{\partial z}: \xi \in U\right\}
$$

consists of all vector fields vanishing at $e$, and

$$
\mathfrak{p}=\mathfrak{p}^{0} \oplus\left\{\left(\xi-\left\{z \xi^{*} z\right\}\right) \frac{\partial}{\partial z}: \xi \in U\right\}
$$

is the tangent space at $e$. 
Denoting by $G$ the connected Lie subgroup of $L$ with Lie algebra $\mathfrak{g}, \mathfrak{l}$ becomes the Lie algebra of the maximal compact subgroup

$$
K=\{g \in G: g(e)=e\}
$$

(which contains nonlinear transformations if $U \neq\{0\}$ ). In the complex case, $G$ is the identity component of the biholomorphic automorphism group of $\Pi$ [L3, U1]. The Lie algebra $\mathfrak{g l}(\Lambda)$ has a representation

$$
\mathfrak{g l}(\Lambda) \ni M \mapsto \widetilde{M} \in \mathfrak{g l}(Z)
$$

such that

$$
\widetilde{M} z_{1}+\left(\widetilde{M} z_{1}\right)^{*}=M\left(z_{1}+z_{1}^{*}\right)
$$

for all $M \in \mathfrak{g l}(\Lambda)$. It follows that $\widetilde{M}$ leaves $\Pi$ invariant. The domain $\Pi$ is also invariant under the translations $\tau_{\xi}(z):=z+\xi$ parametrized by $\xi \in U$.

The vector field

$$
z \frac{\partial}{\partial z}=\left\{e e^{*} z\right\} \frac{\partial}{\partial z} \in \mathfrak{g}^{0} \subset \mathfrak{g}
$$

induces a gradation

$$
\mathfrak{g}=\mathfrak{g}^{1} \oplus \mathfrak{g}^{0} \oplus \mathfrak{g}^{-1},
$$

where

$$
\mathfrak{g}^{\lambda}:=\left\{A \in \mathfrak{g}:\left[z \frac{\partial}{\partial z}, A\right]=\lambda A\right\}
$$

We have

$$
\mathfrak{g}^{1}=\left\{\xi \frac{\partial}{\partial z}: \xi \in U\right\}, \quad \mathfrak{g}^{0}=\left\{M z \frac{\partial}{\partial z}: M \in \mathfrak{g l}(\lambda)\right\}
$$

and

$$
\mathfrak{g}^{-1}=\left\{\{z \xi z\} \frac{\partial}{\partial z}: \xi \in U\right\} .
$$

Now fix a frame $e_{1}, \ldots, e_{r}$ of the Jordan algebra $X$, and consider the Peirce decomposition (3.9) of $X$. Similarly, we have

$$
U=\sum_{1 \leq i \leq j \leq r} \bigoplus U_{i j}
$$

where

$$
U_{i j}=\left\{\xi \in U ;\left\{e_{k} e_{k}^{*} \xi\right\}=\frac{\delta_{i k}+\delta_{j k}}{2} \xi, \forall 1 \leq k \leq r\right\} .
$$

The invariants

$$
c:=\operatorname{dim}_{\mathbb{R}} U_{i i} \quad(1 \leq i \leq r)
$$

and

$$
a:=\operatorname{dim}_{\mathbb{R}} U_{i j} \quad(1 \leq i<j \leq r)
$$

are independent of the frame and are called the characteristic multiplicities of $U$. One can show [N1] that, for irreducible symmetric spaces, (7.8) agrees with (3.10). The subspace $\mathfrak{a} \subset \mathfrak{p}^{0} \subset \mathfrak{p}$ defined in (3.11) is still maximal abelian in $\mathfrak{p}$. 
7.9. Proposition. There is a real root decomposition

$$
\mathfrak{g}=\sum_{i \leq j} \mathfrak{g}_{i j}^{1} \oplus \sum_{i, j} \mathfrak{g}_{i j}^{0} \oplus \sum_{i \leq j} \mathfrak{g}_{i j}^{-1} \oplus \mathfrak{m},
$$

with respect to $\mathfrak{a}$, where $\mathfrak{g}_{i j}^{0}$ and $\mathfrak{m}$ are as in Proposition 3.14, and

$$
\mathfrak{g}_{i j}^{1}:=\left\{\xi \frac{\partial}{\partial z}: \xi \in U_{i j}\right\}
$$

is the root space of

$$
\frac{1}{2}\left(M_{e_{i}}^{\#}+M_{e_{j}}^{\#}\right)
$$

whereas

$$
\mathfrak{g}_{i j}^{-1}:=\left\{\left\{z \xi^{*} z\right\} \frac{\partial}{\partial z}: \xi \in U_{i j}\right\}
$$

is the root space of

$$
-\frac{1}{2}\left(M_{e_{i}}^{\#}+M_{e_{j}}^{\#}\right)
$$

Proof. For $\xi \in U_{i j},(2.1),(2.2)$, and (7.6) imply

$$
\left[\left\{e_{k} e^{*} z\right\} \frac{\partial}{\partial z}, \xi \frac{\partial}{\partial z}\right]=\left\{e_{k} e^{*} \xi\right\} \frac{\partial}{\partial z}=\frac{\delta_{i k}+\delta_{j k}}{2} \xi \frac{\partial}{\partial z}
$$

and

$$
\begin{aligned}
& {\left[\left\{e_{k} e^{*} z\right\} \frac{\partial}{\partial z},\left\{z \xi^{*} z\right\} \frac{\partial}{\partial z}\right]} \\
& \quad=\left(\left\{e_{k} e^{*}\left\{z \xi^{*} z\right\}\right\}-2\left\{\left\{e_{k} e^{*} z\right\} \xi^{*} z\right\}\right) \frac{\partial}{\partial z} \\
& \quad=-\left\{z\left\{e e_{k}^{*} \xi^{*}\right\}^{*} z\right\} \frac{\partial}{\partial z}=-\frac{\delta_{i k}+\delta_{j k}}{2}\left\{z \xi^{*} z\right\} \frac{\partial}{\partial z} .
\end{aligned}
$$

This proves (7.11) and (7.12), and shows that $\mathfrak{g}^{ \pm 1}$ is the direct sum of $\mathfrak{g}_{i j}^{ \pm 1}$. Applying Proposition 3.14, the direct decomposition (7.10) follows. Q.E.D.

We now treat the general case of a (positive definite) Jordan triple $Z$ with a Peirce decomposition $Z=X \oplus U \oplus V$ with respect to a maximal tripotent $e$ (the unit element of $X$ ). The corresponding Cayley transform

$$
\begin{aligned}
g_{e}\left(z_{1}, z_{2}\right) & =\exp \left(\frac{\pi}{4}\left(e+\left\{z e^{*} z\right\}\right) \frac{\partial}{\partial z}\right)\left(z_{1}, z_{2}\right) \\
& =\left(\left(e+z_{1}\right) \circ\left(e-z_{1}\right)^{-1}, \sqrt{2} z_{2} \circ\left(e-z_{1}\right)^{-1}\right)
\end{aligned}
$$

(cf. [L3, U1]) maps $\Omega$ onto the symmetric Siegel domain

$$
\Pi=\{x+\xi+v: 2 x-\Phi(v, v) \in \Lambda\}
$$

associated with the cone $\Lambda$ of $X$ and the hermitian form $\Phi\left(v_{1}, v_{2}\right):=2$ $\left\{v_{1} v_{2}^{*} e\right\}$. In case $U=i X$ and $V$ is complex, $\Pi$ becomes an hermitian symmetric space. 
7.14. Example. Let $r<R$ be integers and consider the matrix space

$$
Z={ }_{r} \mathbb{K}^{R}={ }_{r} \mathbb{K}^{r} \oplus{ }_{r} \mathbb{K}^{(R-r)}
$$

over $\mathbb{K}=\mathbb{R}, \mathbb{C}$ or $\mathbb{H}$ (quaternions), endowed with the Jordan triple product

$$
\left\{z w^{*} z\right\}:=z w^{*} z \text {. }
$$

Writing the elements of $Z$ as $z=\left(z_{1}, z_{2}\right)$ with $z_{1}$ of size $r \times r$ and $z_{2}$ of size $r \times(R-r)$, we obtain a tripotent $e=(1,0)$, and the associated symmetric Siegel domain

$$
\Pi=\left\{\left(z_{1}, z_{2}\right) \in Z: z_{1}+z_{1}^{*}-z_{2} z_{2}^{*} \in \Lambda_{\mathbb{K}}\right\}
$$

belongs to the symmetric matrix cone $\Lambda_{\mathbb{K}}$ (cf. (3.5)) and the hermitian form $\Phi\left(z_{2}, w_{2}\right):=z_{2} w_{2}^{*}$. For example if $r=1$, we obtain

$$
\Pi=\left\{\left(z_{0}, z_{1}, \ldots, z_{n}\right) \in \mathbb{K}^{1+n}: z_{0}+\bar{z}_{0}>z_{1} \bar{z}_{1}+\cdots+z_{n} \bar{z}_{n}\right\} .
$$

For $\mathbb{K}=\mathbb{C}$, we obtain complex hermitian spaces in this way. For example, (7.15) is holomorphically equivalent to the unit ball in $\mathbb{C}^{1+n}$. In order to determine the (semisimple) Lie algebra $\mathfrak{g}$ associated with $\Pi$ we apply the (adjoint) Cayley transformation and obtain for all $x \in X, \xi \in U, \eta \in V$, and $M \in \mathfrak{l}^{0}$

$$
\begin{aligned}
& \operatorname{Ad}\left(g_{e}\right)\left(\left(x-\left\{z x^{*} z\right\}\right) \frac{\partial}{\partial z}\right)=2\left\{x e^{*} z\right\} \frac{\partial}{\partial z}, \\
& \operatorname{Ad}\left(g_{e}\right)\left(\left(\xi-\left\{z \xi^{*} z\right\}-2\left\{\xi e^{*} z\right\}\right) \frac{\partial}{\partial z}\right)=2 \xi \frac{\partial}{\partial z}, \\
& \operatorname{Ad}\left(g_{e}\right)\left(\left(\xi-\left\{z \xi^{*} z\right\}+2\left\{\xi e^{*} z\right\}\right) \frac{\partial}{\partial z}\right)=2\left\{z \xi^{*} z\right\} \frac{\partial}{\partial z} \\
& \operatorname{Ad}\left(g_{e}\right)\left(\left(\eta-\left\{z \eta^{*} z\right\}-2\left\{\eta e^{*} z\right\}+2\left\{e \eta^{*} z\right\}\right) \frac{\partial}{\partial z}\right) \\
& =\sqrt{2}\left(\eta+2\left\{e \eta^{*} z\right\}\right) \frac{\partial}{\partial z}, \\
& \operatorname{Ad}\left(g_{e}\right)\left(\left(\eta-\left\{z \eta^{*} z\right\}+2\left\{\eta e^{*} z\right\}-2\left\{e \eta^{*} z\right\}\right) \frac{\partial}{\partial z}\right) \\
& =\sqrt{2}\left(\left\{z \eta^{*} z\right\}+2\left\{\eta e^{*} z\right\}\right) \frac{\partial}{\partial z}
\end{aligned}
$$

and

$$
\operatorname{Ad}\left(g_{e}\right) M=M .
$$

It follows that $\mathfrak{g}$ has a Cartan decomposition

$$
\mathfrak{g}=\mathfrak{l}+\mathfrak{p},
$$

where

$$
\begin{aligned}
\mathfrak{l}= & \mathfrak{l}^{0} \oplus\left\{\left(\xi+\left\{z \xi^{*} z\right\}\right) \frac{\partial}{\partial z}: \xi \in U\right\} \\
& \oplus\left\{\left(\eta+2\left\{e \eta^{*} z\right\}-\left\{z \eta^{*} z\right\}-2\left\{\eta e^{*} z\right\}\right) \frac{\partial}{\partial z}: \eta \in V\right\}
\end{aligned}
$$


consists of all vector fields vanishing at $(e, 0)$, and

$$
\begin{aligned}
p= & p^{0} \oplus\left\{\left(\xi-\left\{z \xi^{*} z\right\}\right) \frac{\partial}{\partial z}: \xi \in U\right\} \\
& \oplus\left\{\left(\eta+2\left\{e \eta^{*} z\right\}+\left\{z \eta^{*} z\right\}+2\left\{\eta e^{*} z\right\}\right) \frac{\partial}{\partial z}: \eta \in V\right\}
\end{aligned}
$$

is the tangent space at $(e, 0)$. Denoting by $G$ the connected Lie subgroup of $L$ with Lie algebra $\mathfrak{g}, \mathfrak{l}$ becomes the Lie algebra of the maximal compact subgroup

$$
K=\{g \in G g(e, 0)=(e, 0)\} .
$$

In the complex case, $G$ is the identity component of the biholomorphic automorphism group of $\Pi$. The Lie algebra $\mathfrak{g l}(\Lambda)$ has a representation

$$
\mathfrak{g l}(\Lambda) \ni P \mapsto \widetilde{P} \in \mathfrak{g l}(Z)
$$

such that

$$
\widetilde{M} z_{1}+\left(\widetilde{M} z_{1}\right)^{*}-\Phi\left(\widetilde{M} z_{2}, z_{2}\right)-\Phi\left(z_{2}, \widetilde{M} z_{2}\right)=M\left(z_{1}+z_{1}^{*}-\Phi\left(z_{2}, z_{2}\right)\right)
$$

for all $M \in \mathfrak{g l}(\Lambda)$. The domain $\Pi$ is invariant under the "pseudotranslations"

$$
\tau_{\xi, \eta}\left(z_{1}, z_{2}\right):=\left(z_{1}+\xi+\Phi\left(z_{2}, \eta\right)+\frac{\Phi(\eta, \eta)}{2}, z_{2}+\eta\right)
$$

parametrized by $(\xi, \eta) \in U \oplus V$. The vector field

$$
\left\{e e^{*} z\right\} \frac{\partial}{\partial z}=z_{1} \frac{\partial}{\partial z_{1}}+\frac{z_{2}}{2} \frac{\partial}{\partial z_{2}} \in \mathfrak{g}
$$

induces a gradation

$$
\mathfrak{g}=\mathfrak{g}^{1} \oplus \mathfrak{g}^{1 / 2} \oplus \mathfrak{g}^{0} \oplus \mathfrak{g}^{-1 / 2} \oplus \mathfrak{g}^{-1}
$$

into the $\lambda$-eigenspaces $\mathfrak{g}^{\lambda}$ of $\operatorname{ad}\left(\left\{e e^{*} z\right\} \frac{\partial}{\partial z}\right)$. We have

$$
\begin{gathered}
\mathfrak{g}^{1}=\left\{\xi \frac{\partial}{\partial z}: \xi \in U\right\}, \\
\mathfrak{g}^{1 / 2}=\left\{\left(\eta+2\left\{z \eta^{*} e\right\} \frac{\partial}{\partial z}: \eta \in V\right\}, \quad \mathfrak{g}^{0}=\left\{\widetilde{M} z \frac{\partial}{\partial z}: M \in \mathfrak{g l}(\Lambda)\right\},\right. \\
\mathfrak{g}^{-1 / 2}=\left\{\left(\left\{z \eta^{*} z\right\}+2\left\{z e^{*} \eta\right\}\right) \frac{\partial}{\partial z}: \eta \in V\right\},
\end{gathered}
$$

and

$$
g^{-1}=\left\{\left\{z \xi^{*} z\right\} \frac{\partial}{\partial z}: \xi \in U\right\} .
$$

Now fix a frame $e_{1}, \ldots, e_{r}$ of the Jordan algebra $X$ and consider the Peirce decompositions (3.9) and (7.6) of $X$ and $U$, respectively. Similarly, we have

$$
V=\sum_{j=1}^{r} \bigoplus V_{j}
$$

where

$$
V_{j}=\left\{\eta \in V:\left\{e_{k} e_{k}^{*} \eta\right\}=\frac{\delta_{j k}}{2} \eta, \forall 1 \leq k \leq r\right\}
$$


The invariant

$$
b:=\operatorname{dim}_{\mathbb{R}} V_{j} \quad(1 \leq j \leq r)
$$

is independent of the frame and is called the characteristic multiplicity of $V$. For example, in the matrix space $Z={ }_{r} \mathbb{K}^{R}, V_{j}$ consists of all $r \times(R-r)$ matrices $v$ with nonzero entries only in the $j$ th row. The subspace $\mathfrak{a} \subset \mathfrak{p}^{0} \subset \mathfrak{p}$ defined in (3.11) is still maximal abelian in $\mathfrak{p}$.

7.23. Proposition. There is a real root decomposition

$$
\begin{aligned}
\mathfrak{g}= & \sum_{i \leq j} \mathfrak{g}_{i j}^{1} \oplus \sum_{j} \mathfrak{g}_{j}^{1 / 2} \oplus \sum_{i, j} \mathfrak{g}_{i j}^{0} \\
& \oplus \sum_{j} g_{j}^{-1 / 2} \oplus \sum_{i \leq j} g_{i j}^{-1} \oplus m,
\end{aligned}
$$

with respect to $\mathfrak{a}$, where $\mathfrak{g}_{i j}^{ \pm 1}, \mathfrak{g}_{i j}^{0}$ and $\mathfrak{m}$ are as in Propositions 7.9 and 3.14, and

is the root space of

$$
\mathfrak{g}_{j}^{1 / 2}:=\left\{\left(\eta+2\left\{e \eta^{*} z\right\}\right) \frac{\partial}{\partial z}: \eta \in V_{j}\right\}
$$

$$
\frac{1}{2} M_{e_{j}}^{\#}
$$

whereas

$$
\mathfrak{g}_{j}^{-1 / 2}:=\left\{\left(\left\{z \eta^{*} z\right\}+2\left\{\eta e^{*} z\right\}\right) \frac{\partial}{\partial z}: \eta \in V_{j}\right\}
$$

is the root space of

$$
-\frac{1}{2} M_{e_{j}}^{\#}
$$

Proof. For $\eta \in V_{j},(2.1),(2.2)$, and (7.21) imply

$$
\begin{aligned}
& {\left[\left\{e_{k} e^{*} z\right\} \frac{\partial}{\partial z},\left(\eta+2\left\{e \eta^{*} z\right\}\right) \frac{\partial}{\partial z}\right]} \\
& =\left(\left\{e_{k} e^{*} \eta\right\}+2\left(\left\{e_{k} e^{*}\left\{e \eta^{*} z\right\}\right\}-\left\{e \eta^{*}\left\{e_{k} e^{*} z\right\}\right\}\right)\right) \frac{\partial}{\partial z} \\
& =\left(\left\{e_{k} e^{*} \eta\right\}+2\left\{e\left\{e_{k} e^{*} \eta\right\}^{*} z\right\}\right) \frac{\partial}{\partial z} \\
& \left.=\frac{\delta_{j k}}{2}\left(\eta+2 e \eta^{*} z\right\}\right) \frac{\partial}{\partial z}
\end{aligned}
$$

since $\left\{e_{k} \eta^{*} e\right\}=0$. Similarly,

$$
\begin{aligned}
& {\left[\left\{e_{k} e^{*} z\right\} \frac{\partial}{\partial z},\left(\left\{z \eta^{*} z\right\}+2\left\{\eta e^{*} z\right\}\right) \frac{\partial}{\partial z}\right]} \\
& =\left(\left\{e_{k} e^{*}\left\{z \eta^{*} z\right\}\right\}+2\left\{e_{k} e^{*}\left\{\eta e^{*} z\right\}\right\}\right) \frac{\partial}{\partial z} \\
& =\left(\left\{\left\{e_{k} e^{*} z\right\} \eta^{*} z\right\}-\left\{\eta e^{*}\left\{e_{k} e^{*} z\right\}\right\}\right) \frac{\partial}{\partial z} \\
& =-\left(\left\{z\left\{e_{k} e^{*} \eta\right\}^{*} z\right\}+2\left\{\left\{e_{k} e^{*} \eta\right\} e^{*} z\right\}\right) \frac{\partial}{\partial z} \\
& =-\frac{\delta_{i j}}{2}\left(\left\{z \eta^{*} z\right\}+2\left\{\eta e^{*} z\right\}\right) \frac{\partial}{\partial z} .
\end{aligned}
$$


This proves (7.25) and (7.26), and shows that $\mathfrak{g}^{ \pm 1 / 2}$ is the direct sum of $\mathfrak{g}_{j}^{ \pm 1 / 2}$. Applying Propositions 7.9 and 3.14, the direct decomposition (7.24) follows. Q.E.D.

\section{CONICAL AND SPHERICAL FUNCTIONS ON $\Pi$}

We will now construct conical and spherical Laplace eigenfunctions for the symmetric tube and Siegel domains $\Pi$ discussed is Section 7. Endow $\Pi$ with the unique $G$-invariant Riemannian structure such that the basis (3.11) of $\mathfrak{a} \subset$ $\mathfrak{p}^{0} \subset \mathfrak{p} \approx T_{e}(\Pi)$ (tangent space) becomes orthonormal.

Let $N$ be the connected Lie subgroup with Lie algebra

$$
\mathfrak{n}=\mathfrak{g}^{1} \oplus \mathfrak{g}^{1 / 2} \oplus \mathfrak{n}^{0}=\sum_{i \leq j} \mathfrak{g}_{i j}^{1} \oplus \sum_{j} \mathfrak{g}_{j}^{1 / 2} \oplus \sum_{i<j} \mathfrak{g}_{i j}^{0}
$$

(For tube domains, the $\mathfrak{g}^{1 / 2}$-part is missing.) Then there is a (direct) Iwasawa decomposition $[\mathrm{H} 1]$

$$
\Pi=N \cdot \varphi(\mathfrak{a})
$$

where $\varphi$ is defined in (4.1).

Let $\mathscr{D}(\Pi)$ denote the algebra of all (scalar) differential operators $\mathscr{D}$ on $\Pi$ which are invariant under $G$, i.e., satisfy (4.3) for all $f \in \mathscr{C}^{\infty}(\Pi)$ and $g \in G$. For example, the Laplace-Beltrami operator $\Delta$ of $\Pi$ belongs to $\mathscr{D}(\Pi)$. By [H1, Theorem II.3.6], every $D \in \mathscr{D}(\Pi)$ has an $N$-radial part $D_{N}$, which is a differential operator on $\mathfrak{a}$ satisfying (4.4) for all $N$-invariant functions $f \in \mathscr{C}^{\infty}(\Pi)$.

8.2. Proposition. $\Delta$ has the $N$-radial part

$$
\Delta_{N}=\sum_{i}\left(\frac{\partial^{2}}{\partial t_{1}^{2}}-\left(a(i-1)+c+\frac{b}{2}\right) \frac{\partial}{\partial t_{i}}\right) .
$$

Proof. For any $D \in \mathscr{D}(\Pi), D_{N}$ is a constant-coefficient differential operator of the form

$$
D_{N}=q\left(\frac{\partial}{\partial t_{1}}-\rho_{1}, \ldots, \frac{\partial}{\partial t_{r}}-\rho_{r}\right),
$$

where $q$ is any even symmetric (i.e., Weyl group invariant) polynomial, and

$$
\rho_{i}=\frac{a}{2}(i-1)+\frac{c}{2}+\frac{b}{4}
$$

are the coefficients of the half-sum of positive roots

$$
\begin{aligned}
\rho & =\frac{a}{2} \sum_{i<j}\left[\frac{1}{2}\left(M_{e_{j}}^{\#}-M_{e_{i}}^{\#}\right)+\frac{1}{2}\left(M_{e_{j}}^{\#}+M_{e_{i}}^{\#}\right)\right]+\frac{c}{2} \sum_{i} M_{e_{i}}^{\#}+\frac{b}{2} \sum_{i} \frac{1}{2} M_{e_{i}}^{\#} \\
& =\frac{1}{2} \sum_{i}\left(a(i-1)+c+\frac{b}{2}\right) M_{e_{i}}^{\#}
\end{aligned}
$$

(cf. Propositions 7.9 and 7.23). By [H1, Proposition II.3.8], $\Delta$ corresponds to the polynomial

$$
q\left(s_{1}, \ldots, s_{r}\right)=\sum_{i}\left(s_{i}^{2}-\rho_{i}^{2}\right)
$$


so that

$$
\Delta_{N}=\sum_{i}\left(\frac{\partial^{2}}{\partial t_{i}^{2}}-2 \rho_{i} \frac{\partial}{\partial t_{i}}\right) \text {. Q.E.D. }
$$

8.6. Proposition. For each $\alpha \in \mathbb{C}^{r}$, the smooth function

$$
N_{\alpha}(x+\xi+v)=N_{\alpha}\left(x-\frac{\Phi(v, v)}{2}\right)
$$

on $\Pi$ is an $N$-invariant eigenfunction of $\Delta$, with eigenvalue

$$
\chi_{\alpha}=\sum_{i} \alpha_{i}\left(\alpha_{i}-a(i-1)-c-\frac{b}{2}\right) .
$$

Proof. The group $N$ is generated by $N^{0}$ and the pseudo-translations (7.19). Since the expression $2 x-\Phi(v, v)$ is invariant under pseudo-translations and satisfies (7.18) for all $P \in N^{0}$ it follows that (8.7) is $N$-invariant. Since

$$
\left(N_{\alpha} \circ \varphi\right)\left(t_{1}, \ldots, t_{r}\right)=e^{t_{1} \alpha_{1}+\cdots+t_{r} \alpha_{r}},
$$

every $D \in \mathscr{D}(\Pi)$ given by (8.3) satisfies

$$
D N_{\alpha}=q\left(\alpha_{1}-\rho_{1}, \ldots, \alpha_{r}-\rho_{r}\right) N_{\alpha} .
$$

For the polynomial (8.5), we obtain

$$
\Delta N_{\alpha}=\sum_{i}\left(\alpha_{i}^{2}-2 \alpha_{i} \rho_{i}\right) N_{\alpha} \text {. Q.E.D. }
$$

We now pass to the maximal compact subgroup $K$ of $G$. Let

$$
\mathfrak{a}_{+}:=\left\{\sum_{i=1}^{r} t_{i} M_{e_{i}} 0<t_{1}<\cdots<t_{r}\right\}
$$

denote the "Weyl chamber". Then there is a Cartan decomposition

$$
\Pi=K \varphi\left(\mathfrak{a}_{+}\right),
$$

where $\varphi$ is given by (4.1). By [H1, Theorem II.3.6], every $D \in \mathscr{D}(\Pi)$ has a $K$-radial part $D_{K}$ which is a differential operator on $\mathfrak{a}_{+}$satisfying (4.15) for all $K$-invariant functions $f \in \mathscr{C}^{\infty}(\pi)$.

8.11. Proposition. $\Delta$ has the $K$-radial part

$$
\begin{aligned}
\Delta_{K}= & \sum_{i=1}^{r}\left(\frac{\partial^{2}}{\partial t_{i}^{2}}+\left(c \operatorname{coth}\left(t_{i}\right)+b \operatorname{coth}\left(\frac{t_{i}}{2}\right)\right) \frac{\partial}{\partial t_{i}}\right) \\
& +a \sum_{i \neq j} \frac{\sinh \left(t_{i}\right)}{\cosh \left(t_{j}\right)-\cosh \left(t_{i}\right)} \frac{\partial}{\partial t_{i}} .
\end{aligned}
$$

Proof. The positive roots (3.16), (7.11), and (7.25) induce the density function

$$
\begin{aligned}
\delta\left(t_{1}, \ldots, t_{r}\right) & :=\prod_{j} \sinh ^{c}\left(t_{j}\right) \sinh ^{b}\left(\frac{t_{j}}{2}\right) \prod_{i<j} \sinh ^{a}\left(\frac{t_{j}-t_{i}}{2}\right) \sinh ^{a}\left(\frac{t_{j}+t_{i}}{2}\right) \\
& =\prod_{j} \sinh ^{c}\left(t_{j}\right) \sinh ^{b}\left(\frac{t_{j}}{2}\right) \prod_{i<j}\left[\sinh ^{2}\left(\frac{t_{j}}{2}\right)-\sinh ^{2}\left(\frac{t_{i}}{2}\right)\right]^{a} \\
& =\text { const } \cdot \omega^{a} \pi,
\end{aligned}
$$


where

$$
\begin{aligned}
& \omega=\prod_{i<j}\left(\sinh ^{2}\left(\frac{t_{j}}{2}\right)-\sinh ^{2}\left(\frac{t_{i}}{2}\right)\right), \\
& \pi=\prod_{j} \sinh ^{c}\left(t_{j}\right) \sinh ^{b}\left(\frac{t_{j}}{2}\right) .
\end{aligned}
$$

By $[\mathrm{H} 1$, p. 335] we have

$$
\begin{aligned}
\Delta_{K} & =\frac{1}{\delta} \sum_{i} \frac{\partial}{\partial t_{i}} \delta \frac{\partial}{\partial t_{i}}=\frac{1}{\omega^{a} \pi} \sum_{i} \frac{\partial}{\partial t_{i}} \omega^{a} \pi \frac{\partial}{\partial t_{i}} \\
& =\sum_{i}\left(\frac{\partial}{\partial t_{i}}+\frac{1}{\pi} \frac{\partial \pi}{\partial t_{i}}+\frac{a}{\omega} \frac{\partial \omega}{\partial t_{i}}\right) \frac{\partial}{\partial t_{i}} \\
& =\sum_{i}\left(\frac{\partial}{\partial t_{i}}+c \operatorname{coth}\left(t_{i}\right)+b \operatorname{coth}\left(\frac{t_{i}}{2}\right)+a \sum_{j \neq i} \frac{\sinh \left(t_{i}\right)}{\cosh \left(t_{j}\right)-\cosh \left(t_{i}\right)}\right) \frac{\partial}{\partial t_{i}} .
\end{aligned}
$$

Q.E.D.

Now consider the mapping $\psi: \mathfrak{a}_{+} \rightarrow \Lambda \subset \Pi$ defined by

$$
\psi\left(t_{1}, \ldots, t_{r}\right)=\sum_{i=1}^{r} \sinh ^{2}\left(\frac{t_{i}}{2}\right) e_{i}
$$

\subsection{Corollary.}

$$
\begin{aligned}
\psi_{*} \Delta_{K}= & \sum_{i}\left(x_{i}\left(x_{i}+1\right) \frac{\partial^{2}}{\partial x_{i}^{2}}+\left(\frac{1+b+c}{2}+\left(1+c+\frac{b}{2}\right) x_{i}\right) \frac{\partial}{\partial x_{i}}\right) \\
& +a \sum_{i \neq j} \frac{x_{i}\left(x_{i}+1\right)}{x_{j}-x_{i}} \frac{\partial}{\partial x_{i}} .
\end{aligned}
$$

Proof. Putting $x_{i}=\sinh ^{2}\left(\frac{t_{i}}{2}\right)$ we have by (8.13)

$$
\omega=\prod_{i<j}\left(x_{j}-x_{i}\right), \quad \pi=\prod_{i}\left(x_{i}\left(x_{i}+1\right)\right)^{c / 2} x_{i}^{b / 2}
$$

and (8.12) implies

$$
\begin{aligned}
\psi_{*} \Delta_{K}= & \sum_{i}\left(\frac{d^{2} x_{i}}{d t_{i}^{2}}+\left(\frac{d x_{i}}{d t_{i}}\right)^{2}\left(\frac{\partial}{\partial x_{i}}+\frac{1}{\pi} \frac{\partial \pi}{\partial x_{i}}+\frac{a}{\omega} \frac{\partial \omega}{\partial x_{i}}\right)\right) \frac{\partial}{\partial x_{i}} \\
= & \sum_{i}\left(x_{i}+\frac{1}{2}+x_{i}\left(1+x_{i}\right)\left(\frac{\partial}{\partial x_{i}}+\frac{c}{2} \frac{1+2 x_{i}}{x_{i}\left(1+x_{i}\right)}\right.\right. \\
& \left.\left.+\frac{b}{2 x_{i}}+a \sum_{j \neq i} \frac{1}{x_{j}-x_{i}}\right)\right) \frac{\partial}{\partial x_{i}} \text {. Q.E.D. }
\end{aligned}
$$

8.16. Theorem. For each $\alpha \in \overrightarrow{\mathbb{N}}^{r}$, there exists a spherical function $K_{\alpha}$ on $\Pi$ with eigenvalue

$$
\chi_{\alpha}=\sum_{i} \alpha_{i}\left(\alpha_{i}+a(r-i)+c+\frac{b}{2}\right)
$$


which satisfies

$$
K_{\alpha} \circ \varphi \circ \psi^{-1}=\sum_{\beta \leq \alpha} e_{\alpha}^{\beta} K_{\beta}^{0}
$$

Here $\beta$ runs over all partitions dominated by $\alpha$ and the coefficients $c_{\alpha}^{\beta}$ are nonnegative.

Proof. By Corollaries 4.19 and 8.15 we have

$$
\varphi_{*} \Delta_{K}^{0}=D_{2}+\left(1-\frac{a}{2}(r-1)\right) E
$$

and

$$
\psi_{*} \Delta_{K}=D_{2}+D_{1}+\frac{1+b+c}{2} \partial_{e}+\left(1+c+\frac{b}{2}\right) E
$$

where

$$
\begin{aligned}
D_{m} & :=\sum_{i=1}^{r} x_{i}^{m} \frac{\partial^{2}}{\partial x_{i}^{2}}+a \sum_{i \neq j} \frac{x_{i}^{m}}{x_{j}-x_{i}} \frac{\partial}{\partial x_{i}}, \\
\partial_{e} & :=\sum_{i=1}^{r} \frac{\partial}{\partial x_{i}} \quad \text { and } \quad E:=\sum_{i=1}^{r} x_{i} \frac{\partial}{\partial x_{i}} .
\end{aligned}
$$

The commutator identities $\left[\partial_{e}, D_{m}\right]=m D_{m-1}$ and $\left[\partial_{e}, E\right]=\partial_{e}$ imply

$$
\psi_{*} \Delta_{K}=T+\frac{1}{2}\left[\partial_{e}, T\right]+\frac{b}{4} \partial_{e},
$$

where

$$
T:=\varphi_{*} \Delta_{K}^{0}+\left(\frac{a}{2}(r-1)+c+\frac{b}{2}\right) E
$$

Since $K_{\alpha}^{0}$ is homogeneous of degree $|\alpha|:=\alpha_{1}+\cdots+\alpha_{r}$, we have $T K_{\alpha}^{0}=\chi_{\alpha} K_{\alpha}^{0}$, where

$$
\begin{aligned}
\chi_{\alpha} & =\chi_{\alpha}^{0}+\left(\frac{a}{2}(r-1)+c+\frac{b}{2}\right)|\alpha| \\
& =\sum_{i} \alpha_{i}\left(\alpha_{i}+a(r-i)+c+\frac{b}{2}\right) .
\end{aligned}
$$

On the other hand, [Y1] implies

$$
\partial_{e} K_{\alpha}^{0}=\sum_{i}\left(\begin{array}{c}
\alpha \\
\alpha-\varepsilon_{i}
\end{array}\right) K_{\alpha-\varepsilon_{i}}^{0}
$$

with $\alpha-\varepsilon_{i}:=\left(\alpha_{1}, \ldots, \alpha_{i-1}, \alpha_{i}-1, \alpha_{i+1}, \ldots, \alpha_{r}\right)$ and "binomial" coefficients

$$
\left(\begin{array}{c}
\alpha \\
\alpha-\varepsilon_{i}
\end{array}\right) \geq 0
$$


Assuming that $\chi_{\alpha}$ is the $\Delta$-eigenalue of $K_{\alpha}$, we obtain from (8.19)

$$
\begin{aligned}
& \chi_{\alpha} \sum_{\beta} c_{\alpha}^{\beta} K_{\beta}^{0}=\chi_{\alpha}\left(K_{\alpha} \circ \varphi \circ \psi^{-1}\right) \\
& \quad=\left(\Delta K_{\alpha}\right) \circ \varphi \circ \psi^{-1}=\Delta_{K}\left(K_{\alpha} \circ \varphi \circ \psi^{-1}\right) \\
& \quad=\left(\psi_{*} \Delta_{K}\right)\left(K_{\alpha} \circ \varphi \circ \psi^{-1}\right)=\sum_{\beta} c_{\alpha}^{\beta}\left(\psi_{*} \Delta_{K}\right) K_{\beta}^{0} \\
& \quad=\sum_{\beta} c_{\alpha}^{\beta}\left(T+\frac{1}{2} \partial_{e} T-\frac{1}{2} T \partial_{e}+\frac{b}{4} \partial_{e}\right) K_{\beta}^{0} \\
& \quad=\sum_{\beta} c_{\alpha}^{\beta}\left(\chi_{\beta} K_{\beta}^{0}+\frac{1}{2} \sum_{i}\left(\begin{array}{c}
\beta \\
\beta-\varepsilon_{i}
\end{array}\right)\left(\chi_{\beta}-\chi_{\beta-\varepsilon_{i}}+\frac{b}{2}\right) K_{\beta-\varepsilon_{i}}^{0}\right) .
\end{aligned}
$$

Equating coefficients of $K_{\beta}^{0}$, and $\beta<\alpha$ fixed, we obtain

$$
\left(\chi_{\alpha}-\chi_{\beta}\right) c_{\alpha}^{\beta}=\frac{1}{2} \sum_{i}\left(\begin{array}{c}
\beta+\varepsilon_{i} \\
\beta
\end{array}\right)\left(\chi_{\beta+\varepsilon_{i}}-\chi_{\beta}+\frac{b}{2}\right) c_{\alpha}^{\beta+\varepsilon_{i}} .
$$

Since (8.20) implies $\chi_{\alpha}>\chi_{\beta}$ and $\chi_{\beta+\varepsilon_{i}}>\chi_{\beta}$, we may solve for $c_{\alpha}^{\beta}$ inductively, starting with $c_{\alpha}^{\alpha}:=1$, and obtain nonnegative coefficients. Q.E.D.

8.22. Corollary. The spherical function $K_{\alpha}$ on $\Pi$ has a finite expansion

$$
\left(K_{\alpha} \circ \varphi \circ \psi^{-1}\right)(x)=\sum_{\beta \leq \alpha} a_{\alpha}^{\beta} q_{\beta}(x)
$$

in terms of the monomial symmetric functions, with nonnegative coefficients $a_{\alpha}^{\beta} \geq$ 0 .

Proof. Apply Theorem 8.16 and Proposition 4.21. Q.E.D.

9. Stochastic CONTROL THEORY ON SYMMETRIC TUBE OR Siegel DOMAINS:

SPHERICAL FUNCTIONS

A stochastic control problem is formulated in a symmetric tube or Siegel domain $\Pi$ using a $K$-invariance and a spherical polynomial $K_{\alpha}$. The stochastic control problem is the control of a Brownian motion in $\Pi$ by a drift vector field so that this controlled diffusion remains close to the base point $e$ of $\Pi$. Using the $K$-invariance we may use the coordinates $t=\left(t_{1}, \ldots, t_{r}\right)$ for the positive Weyl chamber (8.9).

The controlled diffusion in $\Pi$ has the infinitesimal generator

$$
\frac{1}{2} \Delta+\left\langle u(s, t), \nabla_{t}\right\rangle
$$

where $\Delta$ is the Laplace-Beltrami operator, $t=\left(t_{1}, \ldots, t_{r}\right)$, and $s \in \mathbb{R}_{+}$. Since the $K$-invariance of the control problem is exploited, it suffices to consider the controlled diffusion projected to the positive Weyl chamber $a_{+}$with the infinitesimal generator

$$
\frac{1}{2} \Delta_{K}+\left\langle u(s, t), \nabla_{t}\right\rangle
$$


Let $(X(s), s \geq 0)$ be the controlled diffusion with the infinitesimal generator (9.2) where the dependence on the control $u$ has been suppressed. The cost functional for this stochastic control problem is

$$
J_{\alpha}(u)=E_{X(0)} \int_{0}^{T}\left(K_{\alpha} \circ \psi\right)(X(s))+\langle A(X(s)) U(s, X(s)), U(s, X(s))\rangle d s
$$

where $K_{\alpha}$ is the spherical polynomial (8.23) for the partition $\alpha, A(t):=$ $\operatorname{diag}\left(f_{1}(t), \ldots, f_{r}(t)\right)$,

$$
f_{i}(t)=\frac{1}{{ }_{i} K_{\alpha}(\psi(t))}\left[\frac{\partial}{\partial t_{i}}\left(K_{\alpha} \circ \psi\right)(t)\right]^{2},
$$

and

$$
{ }_{i} K_{\alpha}\left(\sum_{j=1}^{r} x_{j} e_{j}\right)=x_{i} \frac{\partial}{\partial x} \sum_{\mu} \frac{1}{|\mu|} c_{\alpha}^{\mu} q_{\mu}\left(x_{1}, \ldots, x_{r}\right)
$$

for $i=1,2, \ldots, r$. It is clear that

$$
K_{\alpha} \circ \psi=\sum_{i=1}^{r}{ }_{i} K_{\alpha} \circ \psi+{ }_{0} K_{\alpha}
$$

where ${ }_{0} K_{\alpha}$ is a constant.

Since the infinitesimal generator of $(X(s), s \in[0, T])$ is $(9.2)$, the family of stochastic differential equations that $(X(s), s \in[0, T])$ satisfies is

$$
\begin{aligned}
& d X_{k}(s)=\left[\frac{c}{2} \operatorname{coth}\left(X_{k}(s)\right)+\frac{b}{2} \operatorname{coth}\left(\frac{X_{k}(s)}{2}\right)\right. \\
&\left.+\frac{a}{2} \sum_{j \neq k} \frac{\sinh \left(X_{k}(s)\right)}{\cosh \left(X_{k}(s)\right)-\cosh \left(X_{j}(s)\right)}+U(s, X(s))\right] d s=d B_{k}(s)
\end{aligned}
$$

for $k=1,2, \ldots, r, \quad X(0)=\left(X_{1}(0), \ldots, X_{r}(0)\right), X_{1}(0)>X_{2}(0)>$ $\cdots>X_{r}(0)>0, X(t) \in \mathfrak{a}_{+}$, and $\left(B_{1}(s), \ldots, B_{r}(s) ; s \geq 0\right)$ is a standard $r$ dimensional Brownian motion.

Let $(Z(s) \in \Omega, s \in[0, T])$ be the controlled diffusion with the infinitesimal generator $(9.1)$ where the dependence on the control law $U(\cdot)$ has been suppressed for notational simplicity. An admissible control at time $s$ is a Borel measurable function of $Z(s)$ such that the stochastic differential equation whose generator is (9.1) has one and only one strong solution. By the $K$-invariance of the cost functional it suffices to consider controls at time $s$ that are Borel measureable functions of $X(s)$ that satisfy $(9.4)$ so that this family of stochastic differential equations has one and only one strong solution.

Initially it is necessary to verify that the stochastic control problem $(9.1,9.3)$ is well posed, specifically that there is at least one control that gives a finite value to $J_{\alpha}(U)$ in (9.3). The verifcation of the following lemma is the same as the verification of Lemma 6.5 .

9.5. Lemma. Let $(\widetilde{X}(s), s \in[0, T])$ be the diffusion process in $\mathfrak{a}_{+}$with the infinitesimal generator $\frac{1}{2} \Delta_{K}$, that is, the unique strong solution of (9.4) with 
$U(t) \equiv 0$. Then

$$
E_{X(0)} \int_{0}^{T}\left(K_{\alpha} \circ \psi\right)(\tilde{X}(s)) d s<\infty .
$$

The solution of the stochastic control problem $(9.1,9.3)$ is given now.

9.7. Theorem. The stochastic control problem described by $(9.1,9.3)$ has an optimal control $U^{*}$ given by

$$
U^{*}(s, t)=-\frac{g(s)}{2} A^{-1}(t) \nabla_{t}\left(K_{\alpha} \circ \psi\right)(t)
$$

where $s \in[0, T], t=\left(t_{1}, \ldots, t_{r}\right), \nabla_{t}$ is the t-gradient, and $g$ is the unique positive solution of the scalar Riccati differential equation

$$
\frac{d g}{d s}+\frac{1}{2} \chi_{\alpha} g-\frac{1}{4} g^{2}+1=0, \quad g(T)=0,
$$

and $\chi_{\alpha}$ is the eigenvalue (8.17).

Proof. In analogy with the proof of Theorem 6.7, a smooth solution is found for the Hamilton-Jacobi equation for the stochastic optimal control problem of diffusion type. Since the integrand of the cost functional as a function of the state is $K$-invariant, the Hamilton-Jacobi equation can be reduced to the radial part of $(9.1)$ as

$$
0=\frac{\partial W}{\partial s}+\min _{v \in \mathbb{R}^{r}}\left[\frac{1}{2} \Delta_{K}+\sum_{j=1}^{r} v_{j} \frac{\partial W}{\partial t_{j}}+\left(K_{\alpha} \circ \psi\right)(t)+\sum_{j=1}^{r} f_{j} v_{j}^{2}\right] .
$$

It is clear that the family of controls obtained by the minimization in (9.1) is

$$
U_{j}^{*}(s, t)=-\frac{1}{2 f_{j}(t)} \frac{\partial W(s, t)}{\partial t_{j}}
$$

for $j=1,2, \ldots, r$.

Assume a solution $W$ of $(9.10)$ as

$$
W(s, t)=g(s) \sum_{i}\left({ }_{i} K_{\alpha} \circ \psi\right)(t)+h(s) .
$$

Substituting (9.12) into (9.10) it follows that

$$
\begin{aligned}
0= & g^{\prime} \sum_{i} K_{\alpha} \circ \psi+h^{\prime}+\frac{1}{2} \chi_{\alpha} g\left(\sum{ }_{i} K_{\alpha} \circ \psi+{ }_{0} K_{\alpha}\right) \\
& +\sum{ }_{i} K_{\alpha} \circ \psi+{ }_{0} K_{\alpha}-\frac{1}{4} g^{2} \sum{ }_{i} K_{\alpha} \circ \psi \\
= & \sum{ }_{i} K_{\alpha} \circ \psi\left(g^{\prime}+\frac{1}{2} \chi_{\alpha} g-\frac{1}{4} g^{2}+1\right)+h^{\prime}+\frac{1}{2} \chi_{\alpha} g{ }_{0} K_{\alpha}+{ }_{0} K_{\alpha} .
\end{aligned}
$$

If $g$ satisfies (9.9) and $h$ satisfies

$$
h^{\prime}+\frac{1}{2} \chi_{\alpha} g_{0} K_{\alpha}+{ }_{0} K_{\alpha}=0, \quad h(T)=0,
$$

then (9.12) is a smooth solution of (9.10) that satisfies the boundary condition $W(s, t)=0$ for $(s, t) \in\{T\} \times \mathfrak{a}_{+}$. The family of controls $(9.11)$ is an admissible (optimal) control if the family of stochastic differential equations (9.4) with the controls has one and only one strong solution in $[0, T]$. 
Let $(X(s), s \in[0, T])$ be the solution of the family of stochastic differential equations (9.4) with the family of controls (9.11). There is no finite escape time by the methods in the proof of Lemma 6.5. If it is assumed that $(X(s), s \in[0, T])$ does not hit the walls of the positive Weyl chamber then the local smoothness of the coefficients of the stochastic differential equations for $(X(s), s \in[0, T])$ can be used to construct a unique strong global solution (e.g. [IW, p. 164]).

Now it is necessary to verify that $(X(s), s \in[0, T])$ does not hit the walls of the positive Weyl chamber. Initially a subset of a wall away from the origin is investigated. In fact it suffices to consider the process $\left(X_{r-1}(s)-X_{r}(s), s \in\right.$ $[0, T])$ away from the origin because a similar verification is made for the other walls of the Weyl chamber. Choose two positive constants $K \gg c>0$ and define $\Gamma_{r-1}(c, K)$ as

$$
\Gamma_{r-1}(c, K)=\left\{t: K>t_{1}-r c>\cdots>t_{r-2}-3 c>t_{r-1} \geq t_{r}>c\right\} .
$$

By localization by a stopping time it is assumed that $(X(s), s \in[0, T])$ is restricted to $\Gamma_{r-1}(c, K)$.

Let $Y_{r-1}(s)$ be defined as

$$
Y_{r-1}(s)=X_{r-1}(s)-X_{r}(s)
$$

for $s \in[0, T]$. The stochastic differential equation for $\left(Y_{r-1}(s), s \in[0, T]\right)$ is

$$
\begin{aligned}
d Y_{r-1}(s)= & {\left[\frac{a}{2} \frac{\sinh \left(X_{r-1}(s)\right)}{\cosh \left(X_{r-1}(s)\right)-\cosh \left(X_{r}(s)\right)}\right.} \\
& \left.-\frac{a}{2} \frac{\sinh \left(X_{r}(s)\right)}{\cosh \left(X_{r}(s)\right)-\cosh \left(X_{r-1}(s)\right)}+h(s, X(s))\right] d s \\
& +d B_{r-1}(s)-d B_{r}(s)
\end{aligned}
$$

where

$$
\sup _{(s, x) \in[0, T] \times \Gamma_{r-1}(c, K)}|h(s, x)|<M<\infty .
$$

It easily follows from Taylor's formula that there is a $\delta>0$ such that if $0<$ $t_{r-1}-t_{r}<\delta$ then

$$
\frac{a}{2} \frac{\sinh \left(t_{r-1}\right)+\sinh \left(t_{r}\right)}{\cosh \left(t_{r-1}\right)-\cosh \left(t_{r}\right)}-M \geq \frac{1}{t_{r-1}-t_{r}} .
$$

Now localize $(X(s), s \in[0, T])$ to $\Gamma_{r-1}(c, K) \cap\left\{t: t_{r-1}-t_{r}<\delta\right\}$. Apply a comparison theorem [IW, p. 352] for the solutions of scalar stochastic differential equations to the above localization of (9.16) and the so-called two-dimensional Bessel stochastic differential equation

$$
d Z(s)=\frac{1}{Z(s)} d s+\sqrt{2} d B(s) .
$$

Choosing sequences $c_{n} \downarrow 0$ and $K_{n} \uparrow+\infty$ and applying the above construction to $\Gamma_{r-1}\left(c_{n}, K_{n}\right)$ it follows that

$$
P\left(X_{1}(s)>X_{2}(s)>\cdots>X_{r-1}(s)=X_{r}(s)>0 \text { for some } s \in[0, T]\right)=0 \text {. }
$$

Now the behavior of $(X(s), s \in[0, T])$ is investigated near the origin. For this analysis it is more convenient to the coordinates $x=\left(x_{1}, \ldots, x_{r}\right)$ instead 
of $t=\left(t_{1}, \ldots, t_{r}\right)$ where $x_{i}=\sinh ^{2}\left(t_{i} / 2\right)$. Since the radial part of the LaplaceBeltrami operator has been expressed in these coordinates by the chain rule, the family of stochastic differential equations for

$$
Y_{k}(s)=\sinh ^{2} \frac{X_{k}(s)}{2}
$$

for $s \in[0, T]$ is

$$
\begin{aligned}
d Y_{k}(s)= & {\left[\frac{1}{2} Y_{k}(s)\left(1+Y_{k}(s)\right) \vee 0\right]^{1 / 2} d B_{k}(s) } \\
& +\left[\frac{1}{4}(\mathrm{i}+b+c)+\frac{1}{2}\left(1+c+\frac{b}{2}\right) Y_{k}(s)\right. \\
& \left.\quad+\frac{a}{2} \sum_{j \neq k} \frac{Y_{k}(s)\left(1+Y_{k}(s)\right)}{Y_{k}(s)-Y_{j}(s)}-U_{k}^{*}\left(s, \psi^{-1}(Y(s))\right)\right] d s
\end{aligned}
$$

for $k=1,2, \ldots, r$.

Choose two positive numbers $K \gg c>0$ and define $\Lambda_{r}(c, K)$ as

$$
\Lambda_{r}(c, K)=\left\{x: K>x_{1}-r c>\cdots>x_{r-1}-2 c>x_{r}\right\} .
$$

Assume that $(Y(s), s \in[0, T])$ and $(Z(s), s \in[0, T])$ are two pathwise solutions of the family of stochastic differential equations (9.20) that are localized to $\Lambda_{r}(c, K)$. It is elementary to verify that

(9.22)

$$
\left|\left(\frac{1}{2} y(1+y) \vee 0\right)^{1 / 2}-\left(\frac{1}{2} z(1+z) \vee 0\right)^{1 / 2}\right| \leq 2|y-z|^{1 / 2}+2|y-z|:=\rho(|y-z|) .
$$

For $x=\left(x_{1}, \ldots, x_{r}\right)$ and $k \in\{1, \ldots, r\}$ define $\sigma$ and $b_{k}$ as

$$
\sigma\left(x_{k}\right)=\left(\frac{1}{2} x_{k}\left(1+x_{k}\right) \vee 0\right)^{1 / 2}
$$

$$
b_{k}(s, x)=\frac{1}{4}(1+b+c)+\frac{1}{2}\left(1+c+\frac{b}{2}\right) x_{k}+\frac{a}{2} \sum_{j \neq k} \frac{x_{k}\left(1+x_{k}\right)}{x_{k}-x_{j}}-U_{k}^{*}\left(s, \psi^{-1}(x)\right) \text {. }
$$

Following a construction for a scalar stochastic differential equation [IW, p. 168] let $1>a_{1}>a_{2}>\cdots>a_{n}>\cdots>0$ be defined by

$$
\int_{a_{1}}^{1} \rho^{-2}(u) d u=1, \quad \int_{a_{2}}^{1} \rho^{-2}(u) d u=2, \ldots, \quad \int_{a_{n}}^{1} \rho^{-2}(u) d u=n, \ldots,
$$

where $\rho$ is given by (9.22). Clearly $a_{n} \downarrow 0$. Let $\left(\psi_{n}, n \in \mathbb{N}\right)$ be a sequence of continuous functions such that the support of $\psi_{n}$ is contained in $\left(a_{n}, a_{n-1}\right)$,

$$
0 \leq \psi_{n}(u) \leq \frac{2 \rho^{-1}(u)}{n} \text { and } \int_{a_{n}}^{a_{n-1}} \psi_{n}(u) d u=1 .
$$

Define

$$
\varphi_{n}(x) \int_{0}^{|x|} d y \int_{0}^{y} \psi_{n}(u) d u, \quad x \in \mathbb{R}^{1} .
$$

Then $\varphi_{n} \in C^{2}\left(\mathbb{R}^{1}\right),\left|\varphi_{n}^{\prime}\right| \leq 1$, and $\varphi_{n}(x) \uparrow|x|$ as $n \rightarrow \infty$. 
Fix $n \in \mathbb{N}$ and apply Itô's formula to $\varphi_{n}\left(Y_{k}(s)-Z_{k}(s)\right)$ for $k=1,2, \ldots, r$ and add these $r$ equations to obtain

$$
\begin{aligned}
\sum_{k=1}^{r} \varphi_{n}\left(Y_{k}(s)-Z_{k}(s)\right) \\
=\sum_{k=1}^{r} \int_{0}^{s} \varphi_{n}^{\prime}\left(Y_{k}(u)-Z_{k}(u)\right)\left(\sigma\left(Y_{k}(u)\right)-\sigma\left(Z_{k}(u)\right) d B_{k}(u)\right. \\
\quad+\frac{1}{2} \sum_{k=1}^{r} \int_{0}^{s} \varphi_{n}^{\prime}\left(Y_{k}(u)-Z_{k}(u)\right)\left(b_{k}(u, Y(u))-b_{k}(u, Z(u))\right) d u . \\
\quad+\frac{1}{2} \sum_{k=1}^{r} \int_{0}^{s} \varphi_{n}^{\prime \prime}\left(Y_{k}(u)-Z_{k}(u)\right)\left(\sigma\left(Y_{k}(u)\right)-\sigma\left(Z_{k}(u)\right)\right)^{2} d u .
\end{aligned}
$$

Since the expectation of each of the stochastic integrals in (9.25) is zero, it follows that

$$
\begin{aligned}
& E\left[\sum_{k=1}^{r} \varphi_{n}\left(Y_{k}(s)-Z_{k}(s)\right)\right] \\
& \quad=\sum_{k=1}^{r} E \int_{0}^{s} \varphi_{n}^{\prime}\left(Y_{k}(u)-Z_{k}(u)\right)\left(b_{k}(u, Y(u))-b_{k}(u, Z(u))\right) d u \\
& \quad+\frac{1}{2} \sum_{k=1}^{r} E \int_{0}^{s} \varphi_{n}^{\prime \prime}\left(Y_{k}(u)-Z_{k}(u)\right)\left(\sigma\left(Y_{k}(u)\right)-\sigma\left(Z_{k}(u)\right)\right)^{2} d u \\
& =I_{1}(n)+I_{2}(n) .
\end{aligned}
$$

On $\Lambda_{r}(c, K)$ the drift terms $\left(b_{1}, \ldots, b_{r}\right)$ are smooth so that

$$
\begin{aligned}
\left|I_{1}(n)\right| & \leq \sum_{k=1}^{r} E \int_{0}^{s}\left|b_{k}(u, Y(u))-b_{k}(u, Z(u))\right| d u \\
& \leq \sum_{k=1}^{r} E \int_{0}^{s} \tilde{K}|Y(u)-Z(u)| d u
\end{aligned}
$$

and by the construction of $\varphi_{n}$

$$
\begin{aligned}
\left|I_{2}(n)\right| & \leq \frac{1}{2} \sum_{k=1}^{r} \int_{0}^{s} E \frac{2}{n} \rho^{-2}\left(\left|Y_{k}(u)-Z_{k}(u)\right|\right) \rho^{2}\left(\left|Y_{k}(u)-Z_{k}(u)\right|\right) d u \\
& \leq r \frac{s}{n}
\end{aligned}
$$

By passing to the limit we have

$$
E|Y(s)-Z(s)| \leq \int_{0}^{s} r \widetilde{K}|Y(u)-Z(u)| d u
$$

so $Y(s)=Z(s)$ a.s. Choose $\varepsilon>0$ such that $b_{r}(s, x)>0$ for $x \in \Lambda_{r}(c, I) \cap$ $\left\{x:\left|x_{r}\right|<\varepsilon\right\}$ and let

$$
T_{-\varepsilon}=\inf \left\{s: Y_{r}(s)=-\varepsilon\right\}
$$


Let $S<T_{-\varepsilon}$ be such that $Y_{r}(S)<0$ with positive probability. The stochastic differential equation for $\left(Y_{r}(s), s \in[0, T]\right)$ implies that the map $s \mapsto Y_{r}(s)$ on $\left(S, T_{-\varepsilon}\right) 1_{\left\{Y_{r}(S)<0\right\}}$ is increasing which is impossible. Thus

$$
P\left(T_{-\varepsilon} \leq T\right)=0
$$

and

$$
P\left(Y_{1}(s)>\cdots>Y_{r-1}(s)>Y_{r}(s) \geq 0, \quad s \in[0, T]\right)=1 .
$$

Now it is shown that $\left(Y_{r-1}(s), s \in[0, T]\right)$ does not hit the origin. From this verification it easily follows from the previous results that $\left(Y_{k}(s), s \in[0, T]\right)$ does not hit the origin for $k \in\{1, \ldots, r-2\}$. Consider the following stochastic differential equation:

$$
\begin{aligned}
d \widetilde{Y}_{r-1}(s)= & \left(\frac{1}{2} \widetilde{Y}_{r-1}(s)\left(1+\widetilde{Y}_{r-1}(s)\right) \vee 0\right)^{1 / 2} d B_{r-1}(s) \\
& +\left[\frac{1}{4}(1+b+c)+\frac{1}{2}\left(1+c+\frac{b}{2}\right) \tilde{Y}_{r-1}(s)\right] d s
\end{aligned}
$$

By a test for explosions of a one-dimensional diffusion process (e.g., [IW, p. 362]) it follows that

$$
P_{x}\left(\inf _{s<e} \tilde{Y}_{r-1}(s)>0\right)=1
$$

where $e$ is the explostion time. Choose two positive constants $K \gg c>0$ and define $\Lambda_{r-1}(c, K)$ as

$$
\Lambda_{r-1}(c, K)=\left\{x: K>x_{1}-r c>\cdots>x_{r-2}-3 c>x_{r-1} \geq x_{r} \geq 0\right\} .
$$

There is an $\varepsilon>0$ such that on $\Lambda_{r-1}(c, K) \cap\{x: 0 \leq x \leq \varepsilon\}$ the sum of the last two terms in the drift of the stochastic differential equation for $\left(Y_{r-1}(s)\right.$, $s \in[0, T])$ is positive. This solution can be localized to $\Lambda_{r-1}(c, K) \cap$ $\{x: 0 \leq x \leq \varepsilon\}$ and the stochastic differential equation for $\left(Y_{r-1}(s), s \in[0, T]\right)$ can be compared with (9.20). Let $c_{n} \downarrow 0$ and $K_{n} \uparrow+\infty$ and apply the above techniques to $\Lambda_{r-1}\left(c_{n}, K_{n}\right)$ to verify that

$$
P\left(Y_{1}(s)>Y_{2}(s)>\cdots>Y_{r-1}(s)>Y_{r}(s), s \in[0, T]\right)=1 .
$$

This completes the proof. Q.E.D.

\section{Generalization to ABSTRACT ROOT SYSTEMS}

In this section we indicate how the previous results can be generalized, in a somewhat less geometric way, to root systems with "arbitrary" multiplicities (cf. [D1]). Consider Euclidean space $\mathbb{R}^{r}$ with coordinates $t=\left(t_{1}, \ldots, t_{r}\right)$ and consider the "abstract" Weyl chambers (compare with (4.13) and (8.9))

$$
\mathfrak{a}_{+}^{0}:=\left\{t \in \mathbb{R}^{r}: t_{1}>\cdots>t_{r}\right\}
$$

and

$$
\mathfrak{a}_{+}:=\left\{t \in \mathbb{R}^{r}: t_{1}>\cdots>t_{r}>0\right\},
$$

respectively. Consider a density function $W(t)$, the associated volume element

$$
d V(t):=W(t) d t_{1} \cdots d t_{r}
$$


and the "Laplace-Beltrami operator"

$$
\Delta=\frac{1}{w} \sum_{i} \frac{\partial}{\partial t_{i}} W \frac{\partial}{\partial t_{i}}
$$

The density function is specified by

$$
W^{0}=\prod_{1 \leq i<j \leq r} \sinh ^{a}\left(\frac{t_{j}-t_{i}}{2}\right)
$$

and

$$
W=\prod_{i=1}^{r} \sinh ^{c}\left(t_{i}\right) \sinh ^{b}\left(\frac{t_{i}}{2}\right) \prod_{1 \leq i<j \leq r} \sinh ^{a}\left(\frac{t_{j}-t_{i}}{2}\right) \sinh ^{a}\left(\frac{t_{j}+t_{i}}{2}\right),
$$

respectively. Here $a, b, c$ are arbitrary positive constants. Note that for certain integer values of $a, b$, and $c$, we obtain the density functions associated with a symmetric cone or a symmetric domain, respectively (cf. Sections 4 and 8).

We now pass to "algebraic" coordinates $x_{1}, \ldots, x_{r}$ by putting

$$
x_{i}:=\exp \left(t_{i}\right), \quad\left(x_{1}, \ldots, x_{r}\right)=\varphi\left(t_{1}, \ldots, t_{r}\right)
$$

for $(10.1)$ and

$$
x_{i}:=\sinh ^{2}\left(\frac{t_{i}}{2}\right), \quad\left(x_{1}, \ldots, x_{r}\right)=\psi\left(t_{1}, \ldots, t_{r}\right)
$$

for $(10.2)$. Then in both cases the Weyl chamber corresponds to $x_{1}>x_{2}>$ $\cdots>x_{r}>0$. Modifying the proofs of Corollaries 4.19 and 8.15 we obtain

10.8. Proposition. In terms of the algebraic coordinates, the operators (10.3) have the form

$$
\Delta^{0}=\sum_{i=1}^{r} x_{i}^{2} \frac{\partial^{2}}{\partial x_{i}^{2}}+a \sum_{i \neq j} \frac{x_{i}^{2}}{x_{j}-x_{i}} \frac{\partial}{\partial x_{i}}+\sum_{i=1}^{r}\left(1-\frac{a}{2}(r-1)\right) x_{i} \frac{\partial}{\partial x_{i}}
$$

and

$$
\begin{aligned}
\Delta= & \sum_{i=1}^{r} x_{i}\left(x_{i}+1\right) \frac{\partial^{2}}{\partial x_{i}^{2}}+a \sum_{i \neq j} \frac{x_{i}\left(x_{i}+1\right)}{x_{j}-x_{i}} \frac{\partial}{\partial x_{i}} \\
& +\sum_{i=1}^{r}\left(\frac{1+b+c}{2}+\left(1+c+\frac{b}{2}\right) x_{i}\right) \frac{\partial}{\partial x_{i}} .
\end{aligned}
$$

For every partition $\alpha=\left(\alpha_{1}, \ldots, \alpha_{r}\right)$ of integers $\alpha_{1} \geq \alpha_{2} \geq \cdots \geq \alpha_{r} \geq 0$, let

$$
K_{\alpha}^{0}(x)=\mathscr{I}_{\alpha}\left(x, \frac{2}{a}\right) / \mathscr{I}_{\alpha}\left(1^{r}, \frac{2}{a}\right)
$$

denote the "Jack polynomial" (cf. [S1]) for the parameter $2 / a$, normalized by

$$
K_{\alpha}^{0}(1, \ldots, 1)=1 \text {. }
$$

It is known [S1, Theorem 3.1] that $\Delta^{0} K_{\alpha}^{0}=\chi_{\alpha}^{0} K_{\alpha}^{0}$ where

$$
\chi_{\alpha}^{0}=\sum_{i=1}^{r} \alpha_{i}\left(\alpha_{i}+\frac{a}{2}(r+1-2 i)\right) .
$$

If $a$ is an integer coming from a symmetric cone $\Lambda$ (cf. Section 3 ), $K_{\alpha}^{0}$ is (the restriction of) a spherical function on $\Lambda$. 
10.13. Lemma. There is an expansion

$$
K_{\alpha}^{0}(x)=\sum_{\beta \leq \alpha} c_{\alpha}^{\beta} q_{\beta}(x)
$$

where $\beta$ runs over all partitions $\leq \alpha$ in the (reverse) lexicographic order, the $q_{\beta}$ are the symmetric monomial functions [M1] and the coefficients $c_{\alpha}^{\beta}$ (depending on a) are nonnegative.

Proof. Apply [M2, Theorem 6.3] and observe that the coefficients $w(T)$ computed in [M2, (45)] involve only products and quotients of "generalized hooklengths", which are certainly positive. Q.E.D.

Fix a partition $\alpha$ and let $K_{\alpha}^{0}(x)$ be the polynomial (10.11) associated with $\alpha$ which is homogeneous of degree $|\alpha|$. Using the definition of the monomial symmetric functions we have

$$
K_{\alpha}^{0}(x)=\sum a_{\alpha}(\underline{i}) x_{1}^{i_{1}} \cdots x_{r}^{i_{r}}
$$

where $\underline{i}=\left(i_{1}, \ldots, i_{r}\right)$ and $a_{\alpha}(\underline{i})=a_{\alpha}(\underline{j})$ from the definition of the monomial symmetric functions if $\underline{j}$ is a distinct permutation of $\underline{i}$. For $j \in\{1, \ldots, r\}$ define $a_{\alpha}^{j}(\underline{i})$ as

$$
a_{\alpha}^{j}(\underline{i})=\frac{i_{j}}{|\alpha|} a_{\alpha}(\underline{i})
$$

and

$$
{ }_{j} K_{\alpha}^{0}(x)=\sum a_{\alpha}^{j}(\underline{i}) x_{1}^{i_{1}} \cdots x_{r}^{i_{r}}
$$

where the sum is over distinct $r$-tuples such that $a_{\alpha}^{j}(\underline{i}) \neq 0$. Clearly

$$
K_{\alpha}^{0}(x)=\sum_{j=1}^{r}{ }_{j} K_{\alpha}^{0}(x) .
$$

The cost functional for the stochastic control problem is

$$
J_{\alpha}^{0}(U)=E_{X(0)} \int_{0}^{T}\left(K_{\alpha}^{0} \circ \varphi\right)(X(s))+\sum_{j=1}^{r} f_{j}(X(s)) U_{j}^{2}(s) d s
$$

where

$$
f_{j}(t)=\frac{1}{{ }_{j} K_{\alpha}^{0}(\varphi(t))}\left[\frac{\partial}{\partial t_{j}}\left(K_{\alpha}^{0} \circ \varphi\right)(t)\right]^{2} .
$$

The process $(X(s), s \in[0, T])$ is the controlled diffusion in the "Weyl chamber" $\left\{\left(t_{1}, \ldots, t_{r}\right): t_{1}>t_{2}>\cdots>t_{r}\right\}$. The infinitesimal generator of $(X(s), s \in$ $[0, T])$ is

$$
\begin{aligned}
\frac{1}{2} \Delta^{0}+ & \sum_{j=1}^{r} u_{j}(s, t) \frac{\partial}{\partial t_{j}} \\
= & \frac{1}{2} \sum_{k=1}^{r}\left(\frac{\partial^{2}}{\partial t_{k}^{2}}-\frac{a}{2}(r-1) \frac{\partial}{\partial t_{k}}+a \sum_{j \neq k} \frac{1}{1-\exp \left(t_{j}-t_{k}\right)} \frac{\partial}{\partial t_{k}}\right) \\
& +\sum_{k=1}^{r} u_{k}(s, t) \frac{\partial}{\partial t_{k}} .
\end{aligned}
$$


The associated family of stochastic differential equations is

$$
\begin{aligned}
d X_{k}(s)=\left[-\frac{a}{4}(r-1)+\frac{a}{2} \sum_{j \neq k} \frac{1}{1-\exp \left(X_{j}(s)-X_{k}(s)\right)}\right. & \\
& \left.+U_{k}(s, X(s))\right] d s+d B_{k}(s)
\end{aligned}
$$

for $k=1,2, \ldots, r, X(0)=\left(X_{1}(0), \ldots, X_{r}(0)\right), X_{1}(0)>X_{2}(0)>\cdots>$ $X_{r}(0)$, and $\left(B_{1}(s), \ldots, B_{r}(s) ; s \geq 0\right)$ is a standard $r$-dimensional Brownian motion.

An admissible control at time $s$ is a Borel measurable function of $X(s)$ so that (10.20) with this control has one and only one strong solution.

It follows in analogy with Lemma 6.5 that $J_{\alpha}^{0}(0)<\infty$.

The solution of the stochastic optimal control problem $(10.17,10.19)$ is given now.

10.21. Theorem. Assume that $a \geq 1$. The stochastic control problem described by $(10.17,10.19)$ has an optimal control that is

$$
U_{j}^{*}(s, t)=\left[\frac{-{ }_{j} K_{\alpha}^{0}(\varphi(t))}{2 D_{j}\left(K_{\alpha}^{0} \circ \varphi\right)(t)}\right] g(s)
$$

where $j \in\{1, \ldots, r\}, s \in[0, T], t=\left(t_{1}, \ldots, t_{r}\right), D_{j}=\frac{\partial}{\partial t_{j}}$, and $g$ is the unique positive solution of

$$
\frac{d g}{d s}+\frac{1}{2} \chi_{\alpha}^{0} g-\frac{1}{4} g^{2}+1=0, \quad g(T)=0,
$$

where $\chi_{\alpha}^{0}$ is the eigenvalue of $K_{\alpha}^{0}$ for $\Delta^{0}$ given by (10.12).

The proof of this result is not given because it follows closely the proof of Theorem 6.7. Basically it is required to verify that the optimally controlled diffusion does not hit the walls of the "Weyl chamber". Since it is assumed that $a \geq 1$, the inequality (6.20) is satisfied.

In order to study the operator $\Delta$, note that Proposition 10.8 implies

$$
\begin{aligned}
\Delta= & \Delta^{0}+\left(c+\frac{b}{2}+\frac{a}{2}(r-1)\right) E \\
& +\frac{1}{2}\left[\partial_{e}, \Delta^{0}+\left(c+\frac{b}{2}+\frac{a}{2}(r-1)\right) E\right]+\frac{b}{4} \partial_{e},
\end{aligned}
$$

where

$$
E=\sum_{i=1}^{r} x_{i} \frac{\partial}{\partial x_{i}}
$$

is the Euler operator,

$$
\partial_{e}:=\sum_{i=1}^{r} \frac{\partial}{\partial x_{i}},
$$

and $[A, B]:=A B-B A$ is the commutator. This follows as in the proof of Theorem 8.16. As shown in [D1], the operator $\Delta$ has eigenfunctions of the 
form

$$
K_{\alpha}(x)=\sum_{\beta \leq \alpha} a_{\alpha}^{\beta} K_{\beta}^{0}(x)
$$

(called "generalized Jacobi polynomials"). Here $\alpha=\left(\alpha_{1}, \ldots, \alpha_{r}\right)$ is a partition and the coefficients $a_{\alpha}^{\beta}$ depend on $a, b$, and $c$. By [Y1], we have

$$
\partial_{e} K_{\alpha}^{0}=\sum_{i=1}^{r}\left(\begin{array}{c}
\alpha \\
\alpha-\varepsilon_{i}
\end{array}\right) K_{\alpha-\varepsilon_{i}}^{0}
$$

where $\alpha-\varepsilon_{i}:=\left(\alpha_{1}, \ldots, \alpha_{i-1}, \alpha_{i}-1, \alpha_{i+1}, \ldots, \alpha_{r}\right)$ and

$$
\left(\begin{array}{c}
\alpha \\
\alpha-\varepsilon_{i}
\end{array}\right) \geq 0
$$

are the generalized binomial coefficients. Since (10.25) implies that $\partial_{e}$ decreases the "symmetric degree" of a partition, it follows from (10.23) that

$$
\Delta K_{\alpha}=\chi_{\alpha} K_{\alpha}
$$

where

$$
\chi_{\alpha}=\chi_{\alpha}^{0}+\left(\frac{a}{2}(r-1)+c+\frac{b}{2}\right)|\alpha|=\sum_{i} \alpha_{i}\left(\alpha_{i}+a(r-i)+c+\frac{b}{2}\right) .
$$

10.27. Proposition. There is an expansion

$$
K_{\alpha}=\sum_{\beta \leq \alpha} c_{\alpha}^{\beta} q_{\beta}(x)
$$

where the coefficients $c_{\alpha}^{\beta}$ are nonnegative.

Proof. As in the proof of Theorem 8.16, we obtain a recursion formula

$$
2 c_{\alpha}^{\beta} \cdot\left(\chi_{\alpha}-\chi_{\beta}\right)=\sum_{i} c_{\alpha}^{\beta+\varepsilon_{i}}\left(\begin{array}{c}
\beta+\varepsilon_{i} \\
\beta
\end{array}\right) \cdot\left(\chi_{\beta+\varepsilon_{i}}-\chi_{\beta}+\frac{b}{2}\right)
$$

for the coefficients $c_{\alpha}^{\beta}$ of (10.24), starting with $c_{\alpha}^{\alpha}:=1$. Clearly, (10.26) implies

$$
\chi_{\alpha}-\chi_{\beta}>0 \text { and } \chi_{\beta+\varepsilon_{i}}-\chi_{\beta}+\frac{b}{2} \geq 0 .
$$

Therefore $c_{\alpha}^{\beta} \geq 0$ for all $\beta \leq \alpha$. Now apply Lemma 10.13. Q.E.D.

Fix a partition and let $K_{\alpha}(x)$ be the polynomial (10.28). Using the definition of the monomial symmetric functions we have

$$
K_{\alpha}(x)=\sum a_{\alpha}(\underline{i}) x_{1}^{i_{1}} \cdots x_{r}^{i_{r}}
$$

where $\underline{i}=\left(i_{1}, \ldots, i_{r}\right)$ and the sum is over distinct $r$-tuples such that $a_{\alpha}^{j}(\underline{i}) \neq 0$. For $j \in\{1, \ldots, r\}$ define $a_{\alpha}^{j}(\underline{i})$ as

$$
a_{\alpha}^{j}(\underline{i})=\frac{i_{j}}{\sum_{k=1}^{r} i_{k}} a_{\alpha}(\underline{i})
$$

and

$$
{ }_{j} K_{\alpha}(x)=\sum a_{\alpha}^{j}(\underline{i}) x_{1}^{i_{1}} \cdots x_{r}^{i_{r}}
$$


where the sum is over distinct $r$-tuples such that $a_{\alpha}^{j}(\underline{i}) \neq 0$. Clearly

$$
K_{\alpha}(x)=\sum_{j=1}^{r}{ }_{j} K_{\alpha}(x)+{ }_{0} K_{\alpha},
$$

where ${ }_{0} K_{\alpha}$ is the constant term in $K_{\alpha}$.

The cost functional for the stochastic control problem is

$$
J_{\alpha}(U)=E_{X(0)} \int_{0}^{T}\left(K_{\alpha} \circ \psi\right)(X(s))+\sum_{j=1}^{r} f_{j}(X(s)) U_{j}^{2}(s) d s
$$

where

$$
f_{j}(t)=\frac{1}{{ }_{j} K_{\alpha}(\psi(t))}\left[\frac{\partial}{\partial t_{j}}\left(K_{\alpha} \circ \psi\right)(t)\right]^{2} .
$$

The process $(X(s), s \in[0, T])$ is the controlled diffusion in the "Weyl chamber" $\left\{\left(t_{1}, \ldots, t_{r}\right): t_{1}>t_{2}>\cdots>t_{r}>0\right\}$. The infinitesimal generator of $(X(s), s \in[0, T])$ is

$$
\begin{aligned}
\frac{1}{2} \Delta+ & \sum_{j=1}^{r} u_{j}(s, t) \frac{\partial}{\partial t_{j}} \\
= & \sum_{k=1}^{r} \frac{1}{2} \frac{\partial^{2}}{\partial t_{k}^{2}}+\left[\frac{c}{2} \operatorname{coth} t_{k}+\frac{b}{2} \operatorname{coth} \frac{t_{k}}{2}\right] \frac{\partial}{\partial t_{k}} \\
& +\frac{a}{2} \sum_{j \neq k} \frac{\sinh \left(t_{k}\right)}{\cosh \left(t_{j}\right)-\cosh \left(t_{k}\right)} \frac{\partial}{\partial t_{k}} \\
& +\sum_{k=1}^{r} u_{k}(s, t) \frac{\partial}{\partial t_{k}} .
\end{aligned}
$$

The associated family of stochastic differential equations is (10.35)

$$
\begin{aligned}
d X_{k}(s)= & {\left[\frac{b}{2} \operatorname{coth} \frac{X_{k}(s)}{2}+\frac{c}{2} \operatorname{coth} X_{k}(s)\right.} \\
& \left.+\frac{a}{2} \sum_{j \neq k} \frac{\sinh X_{k}(s)}{\cosh \left(X_{j}(s)\right)-\cosh \left(X_{k}(s)\right)}=U_{k}(s, X(s))\right] d s+d B_{k}(s)
\end{aligned}
$$

for $k=1,2, \ldots, r, X(0)=\left(X_{1}(0), \ldots, X_{r}(0)\right), X_{1}(0)>X_{2}(0)>\cdots>$ $X_{r}(0)>0$, and $\left(B_{1}(s), \ldots, B_{r}(s) ; s \geq 0\right)$ is a standard $r$-dimensional Brownian motion.

An admissible control at time $s$ is a Borel measurable function of $X(s)$ so that (10.35) with this control has one and only one strong solution.

It follows in analogy with Lemma 9.5 that $J_{\alpha}(0)<\infty$.

The solution of the stochastic optimal control problem $(10.32,10.34)$ is given now.

10.36. Theorem. Assume that $a \geq 1$. The stochastic control problem described by $(10.32,10.34)$ has an optimal control that is

$$
U_{j}^{*}(s, t)=\left[\frac{-{ }_{j} K_{\alpha}(\psi(t))}{2 D_{j}\left(K_{\alpha} \circ \psi\right)(t)}\right] g(s)
$$


where $j \in\{1, \ldots, r\}, s \in[0, T], t=\left(t_{1}, \ldots, t_{r}\right), D_{j}=\frac{\partial}{\partial t_{j}}$, and $g$ is the unique positive solution of

$$
\frac{d g}{d s}+\frac{1}{2} \chi_{\alpha} g-\frac{1}{4} g^{2}+1=0, \quad g(T)=0,
$$

where $\chi_{\alpha}$ is the eigenvalue of $K_{\alpha}$ for $\Delta$ given by (10.26).

The proof of this result is not given because it follows closely the proof of Theorem 9.7. Basically it is required to verify that the optimally controlled diffusion does not hit the walls $\left\{t_{i}=t_{i-1}\right\}$ for $i=1, \ldots, r$ of the "Weyl chamber". Since it is assumed that $a \geq 1$, the inequality (9.17) is satisfied. The behavior of this diffusion near the origin follows as in the proof of Theorem 9.7.

\section{REFERENCES}

[BK] H. Braun and M. Koecher, Jordan-Algebren, Springer, 1966.

[D1] A. Debiard, Système differentiel hypergéométrique et parties radiales des opérateurs invariants des espaces symétriques de type $B C_{p}$, Lecture Notes in Math., vol. 1296, Springer, 1988, pp. 42-124.

[D2] T. E. Duncan, Stochastic systems in Riemannian manifolds, J. Optim. Theory Appl. 27 (1979), 399-4210.

[D3] - Dynamic programming optimality criteria for stochastic systems in Riemannian manifolds, Appl. Math. Optim. 3 (1977), 191-208.

[D4] $\quad$ A solvable stochastic control problem in hyperbolic three space, Systems Control Lett. 8 (1987), 435-439.

[D5] - Some solvable stochastic control problems in noncompact symmetric spaces of rank one, Stochastics Stochastics Rep. 35 (1991), 129-142.

[DU] T. E. Duncan and H. Upmeier, Stochastic control problems in symmetric cones and spherical functions, Diffusion Processes and Related Problems in Analysis, Vol. 1, Birkhäuser, 1990, pp. 263-283.

[FK] J. Faraut and A. Korányi, Analysis on symmetric cones (to appear).

[F1] R. H. Farrell, Multivariate calculation, Springer-Verlag, New York-Berlin, 1985.

[FR] W. H. Fleming and R. W. Rishel, Deterministic and stochastic optimal control, SpringerVerlag, 1975.

[H1] S. Helgason, Groups and geometric analysis, Academic Press, 1984.

[H2] B. Hoogenboom, Spherical functions and differential operators in complex Grassmann manifolds, Ark. Mat. 29 (1982), 69-85.

[H3] L. Hörmander, The analysis of linear partial differential equations, Vol. I, Springer, 1983.

[IW] N. Ikeda and S. Watanabe, Stochastic differential equations and diffusion processes, NorthHolland, 1981.

[J1] K. D. Johnson, On a ring of invariant polynomials on a Hermitian symmetric space, J. Algebra 67 (1980), 72-81.

[J2] A. T. James, Calculations of zonal polynomial coefficients by use of the Laplace-Beltrami operator, Ann. Math. Statist. 39 (1968), 1711-1718.

[KW] A. Korányi and J. A. Wolf, Generalized Cayley transformations of bounded symmetric domains, Amer. J. Math. 87 (1965), 899-939.

[L1] O. Loos, Symmetric spaces, I, II, Benjamin, 1969.

[L2] _ Jordan triple systems, R-spaces and bounded symmetric domains, Bull. Amer. Math. Soc. 77 (1971), 558-561.

[L3] _ Bounded symmetric domains and Jordan pairs, Univ. of California, Irvine, 1977. 
[M1] I. Macdonald, Symmetric functions and Hall polynomials, Clarendon Press, Oxford, 1979.

[M2] - Commuting differential operators and zonal spherical functions, Lecture Notes in Math., vol. 1271, Springer, 1987, pp. 189-199.

[M2] R. J. Muirhead, Aspects of multivariate statistical theory, Wiley, New York, 1982.

[N1] E. Neher, Klassifikation der einfachen reellen Jordan-Tripelsysteme, Dissertation, Münster, 1978.

[S1] R. P. Stanley, Some combinatorial properties of Jack symmetric functions, Adv. Math. 77 (1989), 76-115.

[T1] A. Takemura, Zonal polynomials, Inst. Math. Stat. Lecture Notes-Monograph Series, 4, 1984.

[U1] H. Upmeier, Symmetric Banach manifolds and Jordan $C^{*}$-algebras, North-Holland, 1985.

[U2] _ Jordan algebras and harmonic analysis on symmetric spaces, Amer. J. Math. 108 (1986), 1-25.

[U3] - Jordan algebras in analysis, Operator Theory and Quantum Mechanics, CMBS Regional Conf. Ser. in Math., no. 67, Providence, RI, 1987.

[Y1] Z. Yan, Hypergeometric functions in several variables, Thesis, CUNY, 1990.

[Y2] K. Yosida, Functional analysis, Springer, 1971.

Department of Mathematics, University of Kansas, Lawrence, Kansas 66045

E-mail address: duncan@kuhub.cc.ukans.edu

Department of Mathematics, University of Marburg, Hans Meerwein Strasse, 35032 MARBURG, GERMANY 